\title{
PROPERTIES OF 42 SOLAR-TYPE KEPLER TARGETS FROM THE ASTEROSEISMIC MODELING PORTAL
}

\author{
T. S. Metcalfe ${ }^{1,2}$, O. L. Creeney ${ }^{3}$, G. DoĞan ${ }^{2,4}$, S. Mathur ${ }^{1,4}$, H. Xu ${ }^{5}$, T. R. Bedding ${ }^{6}$, W. J. Chaplin ${ }^{7}$, \\ J. Christensen-DalsgaArd ${ }^{2}$, C. KarofF ${ }^{2}$, R. Trampedach ${ }^{2,8}$, O. Benomar $^{6,9}$, B. P. Brown ${ }^{10,11}$, D. L. BuZasi ${ }^{12}$, \\ T. L. Campante ${ }^{7}$, Z. Çelik ${ }^{13}$, M. S. Cunha ${ }^{14}$, G. R. Davies ${ }^{7}$, S. Deheuvels ${ }^{15}, 16$, A. Derekas ${ }^{17,18}$, M. P. Di Mauro ${ }^{19}$, \\ R. A. García ${ }^{20}$, J. A. GuziK ${ }^{21}$, R. Howe ${ }^{7}$, K. B. MacGregor ${ }^{4}$, A. Mazumdar ${ }^{22}$, J. Montalbán ${ }^{23}$, M. J. P. F. G. Monteiro ${ }^{14}$, \\ D. Salabert ${ }^{20}$, A. Serenelli ${ }^{24}$, D. Stello ${ }^{6}$, M. StȩŚlicki $^{25}$, M. D. Suran ${ }^{26}$, M. Yildiz ${ }^{13}$, C. Aksoy ${ }^{13}$, Y. Elsworth ${ }^{7}$, \\ M. Gruberbauer ${ }^{27}$, D. B. Guenther ${ }^{27}$, Y. Lebreton ${ }^{28,29}$, K. Molaverdikhani ${ }^{30}$, D. Pricopi ${ }^{26}$, \\ R. SimONiELlo ${ }^{31}$, AND T. R. White ${ }^{6,32}$ \\ ${ }^{1}$ Space Science Institute, 4750 Walnut Street Suite 205, Boulder, CO 80301, USA \\ ${ }^{2}$ Stellar Astrophysics Centre, Department of Physics and Astronomy, Aarhus University, Ny Munkegade 120, DK-8000 Aarhus C, Denmark \\ ${ }^{3}$ Institut d'Astrophysique Spatiale, Université Paris XI, UMR 8617, CNRS, Batiment 121, F-91405 Orsay Cedex, France \\ ${ }^{4}$ High Altitude Observatory, NCAR, P.O. Box 3000, Boulder, CO 80307, USA \\ ${ }^{5}$ Computational \& Information Systems Laboratory, NCAR, P.O. Box 3000, Boulder, CO 80307, USA \\ ${ }^{6}$ Sydney Institute for Astronomy (SIfA), School of Physics, University of Sydney, NSW 2006, Australia \\ ${ }^{7}$ School of Physics and Astronomy, University of Birmingham, Birmingham B15 2TT, UK \\ 8 JILA, University of Colorado and National Institute of Standards and Technology, 440 UCB, Boulder, CO 80309, USA \\ ${ }^{9}$ Department of Astronomy, The University of Tokyo, Tokyo 113-0033, Japan \\ ${ }^{10}$ Department of Astronomy and Center for Magnetic Self Organization in Laboratory and Astrophysical Plasmas, \\ University of Wisconsin, Madison, WI 53706, USA \\ ${ }^{11}$ Kavli Institute for Theoretical Physics, University of California, Santa Barbara, CA 93106, USA \\ ${ }^{12}$ Department of Chemistry and Physics, Florida Gulf Coast University, Fort Myers, FL 33965, USA \\ ${ }^{13}$ Ege University, Department of Astronomy and Space Sciences, Bornova, 35100, Izmir, Turkey \\ ${ }^{14}$ Centro de Astrofísica e Faculdade de Ciências, Universidade do Porto, Rua das Estrelas, 4150-762, Porto, Portugal \\ ${ }^{15}$ Université de Toulouse, UPS-OMP, IRAP, Toulouse, France \\ ${ }^{16}$ CNRS, IRAP, 14, avenue Edouard Belin, F-31400 Toulouse, France \\ ${ }^{17}$ Konkoly Observatory, MTA CSFK, H-1121 Budapest, Konkoly Thege M. út 15-17, Hungary \\ ${ }^{18}$ ELTE Gothard Astrophysical Observatory, H-9704 Szombathely, Szent Imre herceg út 112, Hungary \\ ${ }^{19}$ INAF-IAPS Istituto di Astrofisica e Planetologia Spaziali, Via del Fosso del Cavaliere 100, I-00133 Roma, Italy \\ ${ }^{20}$ Laboratoire AIM, CEA/DSM-CNRS-Univ. Paris Diderot-IRFU/SAp, Centre de Saclay, F-91191 Gif-sur-Yvette Cedex, France \\ ${ }^{21}$ Los Alamos National Laboratory, XTD-NTA, MS T-086, Los Alamos, NM 87545, USA \\ ${ }^{22}$ Homi Bhabha Centre for Science Education, TIFR, V. N. Purav Marg, Mankhurd, Mumbai 400088, India \\ ${ }^{23}$ Institut d'Astrophysique et Geophysique, University of Liege, Belgium \\ ${ }^{24}$ Institute of Space Sciences (CSIC-IEEC), Campus UAB, E-08193, Bellaterra, Spain \\ ${ }^{25}$ Space Research Center, Polish Academy of Sciences, Wrocław, Poland \\ ${ }^{26}$ Astronomical Institute of the Romanian Academy, Str. Cutitul de Argint 5, RO-040557, Bucharest, Romania \\ ${ }^{27}$ Institute for Computational Astrophysics, Department of Astronomy and Physics, Saint Mary's University, Halifax, N.S., B3H 3C3, Canada \\ ${ }^{28}$ Observatoire de Paris, GEPI, CNRS UMR 8111, F-92195, Meudon, France \\ ${ }^{29}$ Institut de Physique de Rennes, Universit de Rennes 1, CNRS UMR 6251, F-35042, Rennes, France \\ ${ }^{30}$ Laboratory for Atmospheric and Space Physics, University of Colorado, Boulder, CO 80309, USA \\ ${ }^{31}$ Laboratoire AIM, CEA/DSM-CNRS-Universit Paris Diderot, IRFU/SAp, Centre de Saclay, F-91191, Gif-sur-Yvette, France \\ 32 Institut für Astrophysik, Georg-August-Universität Göttingen, Friedrich-Hund-Platz 1, D-37077 Göttingen, Germany \\ Received 2014 February 14; accepted 2014 September 2; published 2014 October 1
}

\begin{abstract}
Recently the number of main-sequence and subgiant stars exhibiting solar-like oscillations that are resolved into individual mode frequencies has increased dramatically. While only a few such data sets were available for detailed modeling just a decade ago, the Kepler mission has produced suitable observations for hundreds of new targets. This rapid expansion in observational capacity has been accompanied by a shift in analysis and modeling strategies to yield uniform sets of derived stellar properties more quickly and easily. We use previously published asteroseismic and spectroscopic data sets to provide a uniform analysis of 42 solar-type Kepler targets from the Asteroseismic Modeling Portal. We find that fitting the individual frequencies typically doubles the precision of the asteroseismic radius, mass, and age compared to grid-based modeling of the global oscillation properties, and improves the precision of the radius and mass by about a factor of three over empirical scaling relations. We demonstrate the utility of the derived properties with several applications.
\end{abstract}

Key words: methods: numerical - stars: evolution - stars: interiors - stars: oscillations

Online-only material: color figures, extended figure

\section{BACKGROUND}

It is difficult to overstate the impact of the Kepler mission on the observation and analysis of solar-like oscillations in mainsequence and subgiant stars. In a review from just a decade ago, Bedding \& Kjeldsen (2003) highlighted the tentative detections of individual oscillation frequencies in just a few such stars from ground-based observations, and Kepler was not even mentioned. Despite funding issues that delayed the mission from an original deployment date in 2006, Kepler finally launched in March 2009 and operated almost flawlessly for more than four years, slightly exceeding its design lifetime (Borucki et al. 2010). The archive 
of public data now includes nearly uninterrupted observations for many thousands of solar-type stars, including short-cadence data (58.85 s sampling; Gilliland et al. 2010) for hundreds of these targets. In the span of a decade, the study of solarlike oscillations has been transformed dramatically (Chaplin \& Miglio 2013).

During the first 10 months of science operations, Kepler performed a survey for solar-like oscillations in more than 2000 main-sequence and subgiant stars, yielding detections in more than 500 targets from the one month data sets. The initial analysis of this ensemble, using empirical scaling relations (Kjeldsen \& Bedding 1995) to generate estimates of radius and mass, suggested a significant departure from the mass distribution expected from Galactic population synthesis models (Chaplin et al. 2011). Subsequent analysis of the sample, using updated effective temperatures (Pinsonneault et al. 2012) and a substantial grid-based modeling effort, led to more precise estimates of the radii and masses as well as information about the stellar ages (Chaplin et al. 2014). Some of the brightest stars from the survey were subjected to a more detailed analysis, including spectroscopic follow-up to determine more precise atmospheric properties (Bruntt et al. 2012) plus the identification and detailed modeling of dozens of oscillation frequencies in each star (Mathur et al. 2012). These studies gave us a preview of what to expect from the subsequent phase of the mission.

Starting with Quarter 5 (Q5), Kepler's short-cadence study of solar-like oscillations transitioned to a specific target phase, where extended observations began for a fixed sample of stars identified during the survey. The target list during this phase gave priority to stars showing oscillations with the highest signalto-noise ratio $(\mathrm{S} / \mathrm{N})$, but it also retained the brightest mainsequence stars cooler than the Sun, where the lower intrinsic oscillation amplitudes yielded relatively weak detections from the survey. From Q5 through the end of the mission (Q17), about 200 of the 512 available short-cadence targets were typically specified by the Kepler Asteroseismic Science Consortium (KASC; Kjeldsen et al. 2010) and about half of those were intended for the study of solar-like oscillations.

Just like the exoplanet side of the mission, the KASC team gradually improved the data reduction and analysis methods while additional data swelled the archive. Never in the history of the field had such extended monitoring been possible at all, let alone for such a large sample of stars. As a consequence, the availability of reliable sets of input data for stellar modeling lagged well behind the continually expanding time series for each star in the archive. This delay was primarily due to the challenge of coordinating the efforts of multiple teams, first to produce optimized light curves from the raw Kepler data (García et al. 2011), then to fit the global oscillation properties and remove the stellar granulation background from the power spectra (Verner et al. 2011; Mathur et al. 2011), and finally to extract and identify the individual oscillation frequencies using so-called "peak-bagging" techniques (Appourchaux et al. 2012). Also like the exoplanet program, ground-based follow up observations were difficult to obtain for the faintest targets, further limiting the number of stars for which detailed modeling was feasible.

Even after reliable sets of observational constraints became available, an analogous effort was required to consolidate the results from many stellar modeling teams. Initially this effort sought to define objective metrics of model quality, and to use the ensemble of results from different codes and methods to estimate the systematic uncertainties for a few specific targets
(Metcalfe et al. 2010, 2012; Creevey et al. 2012; Dogan et al. 2013; Silva Aguirre et al. 2013). The first large sample to emerge from the survey made this "boutique" modeling approach impractical, and motivated the initial large-scale application of the Asteroseismic Modeling Portal (AMP; Metcalfe et al. 2009; Woitaszek et al. 2009). Mathur et al. (2012) presented a uniform analysis of 22 Kepler targets observed for 1 month each during the survey phase, and compared detailed modeling from AMP with empirical scaling relations and with results from several grid-based modeling methods that matched the global oscillation properties ( $\Delta v$ and $v_{\max }$, see below) rather than the individual frequencies from peak-bagging. The results clearly demonstrated the improved level of precision that was possible from detailed modeling of the individual oscillation frequencies, particularly for stellar ages.

In this paper, we present stellar modeling results from AMP for the first large sample of Kepler targets with extended observations during the specific target phase of the mission. In Section 2, we describe the sample, which was drawn from the most recently published observations. We outline our stellar modeling approach in Section 3, including several improvements to the previous version of AMP and using slightly customized procedures for different types of stars. We present the main results and initial applications in Section 4, and we discuss conclusions and future prospects in Section 5.

\section{OBSERVATIONAL CONSTRAINTS}

Solar-like oscillations are stochastically excited and intrinsically damped by turbulent convection near the stellar surface (Goldreich \& Keeley 1977; Goldreich \& Kumar 1988; Houdek et al. 1999; Samadi \& Goupil 2001). Each oscillation mode is characterized by its radial order $n$ and spherical degree $\ell$, and only the low-degree $(\ell \leqslant 3)$ modes are generally detectable without spatial resolution across the surface. The consecutive radial orders define the average large separation $\langle\Delta v\rangle$, which reflects the mean stellar density (Tassoul 1980). The power in each mode is governed by a roughly Gaussian envelope with a maximum at frequency $v_{\max }$, which approximately scales with the acoustic cutoff frequency (Brown et al. 1991; Belkacem et al. 2011). These global oscillation properties are well-constrained, even in the relatively short time series obtained during the Kepler survey phase. Longer observations improve the frequency precision and also reveal additional oscillation modes, with lower and higher radial orders, as the $\mathrm{S} / \mathrm{N}$ improves in the wings of the Gaussian envelope. This is the primary motivation for gathering extended observations: to maximize both the number and quality of asteroseismic constraints that are available for stellar modeling.

Appourchaux et al. (2012) published asteroseismic data sets for 61 main-sequence and subgiant stars observed by Kepler, based on an analysis of 9-month time series. The data were collected during Q5-Q7 (2010 March 20 through 2010 December 22), archived on 2011 April 23, and the final sets of identified frequencies were published about one year later. Most of these frequency sets came from maximum likelihood estimators (Appourchaux et al. 2008) with errors determined from the inverse of the Hessian matrix, but frequencies for the F-like stars were obtained from a Bayesian analysis with MultiNest (Gruberbauer et al. 2009) and errors were estimated from the $68 \%$ credible interval. When asymmetric errors were provided, we adopted the mean of the two quoted values. No similar analysis of more extended data sets has yet been 


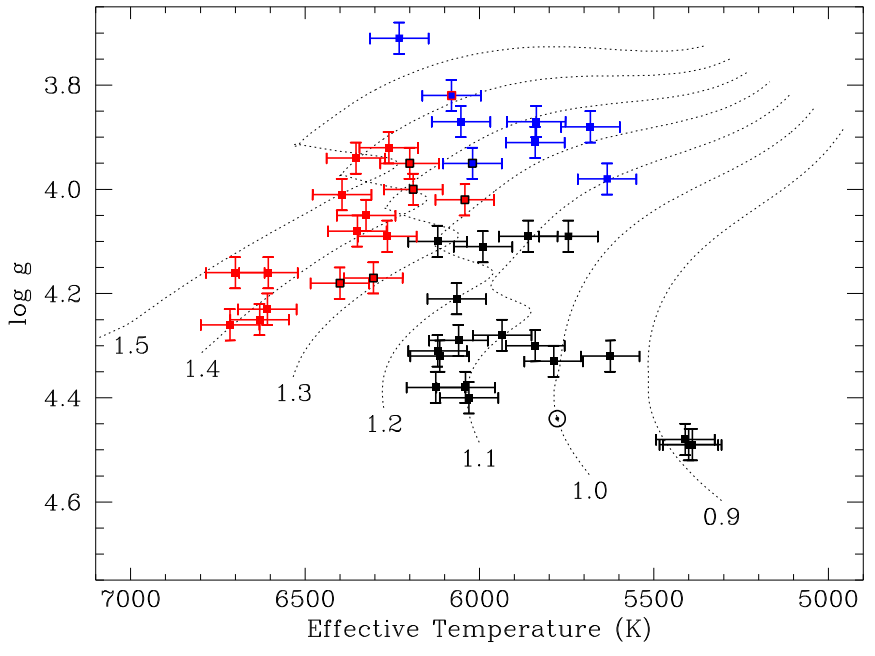

Figure 1. Spectroscopic H-R diagram for our final sample of 42 asteroseismic targets, including simple (black), F-like (red), and mixed-mode stars (blue) Points outlined in a different color indicate the original classification by Appourchaux et al. (2012). Error bars were adopted from Chaplin et al. (2014). Solar-composition evolution tracks from ASTEC for masses between 0.9 and $1.5 M_{\odot}$ are shown as dotted lines, with the position of the Sun indicated by the $\odot$ symbol. Stars similar to the Sun are missing from the KASC sample because the data were sequestered by the Kepler exoplanet team.

(A color version of this figure is available in the online journal.)

published, so we adopted this sample of 61 stars as our uniform source of asteroseismic constraints.

In addition to the asteroseismic data, precise spectroscopic constraints on the effective temperature $T_{\text {eff }}$ and metallicity $[\mathrm{M} / \mathrm{H}]$ are also required for detailed stellar modeling. A uniform spectroscopic analysis of 93 solar-type Kepler targets was published by Bruntt et al. (2012), including 46 of the brightest stars in the Appourchaux et al. sample with magnitudes in the Kepler bandpass $K p=7.4-9.8$. Six of the 15 missing targets are also in this magnitude range: KIC 3735871, 11772920, 12317678 , and 12508433, as well as the two bright spectroscopic binaries KIC 8379927 and 9025370. The remaining 9 stars fall in the magnitude range $K p=9.9-11.4$ and are difficult targets for high-resolution spectroscopy on all but the largest telescopes.

Recently, Molenda-Żakowicz et al. (2013) published spectroscopic constraints for a larger sample of 169 stars in the Kepler field. The overlap with the Appourchaux et al. sample only includes two additional stars (KIC 11772920 and 12508433) for the low-precision ROTFIT results (Frasca et al. 2003, 2006), while the high-precision ARES+MOOG results (Sousa et al. 2007; Sneden 1973) contain fewer asteroseismic targets than are available in the Bruntt et al. sample. Considering that our goal is to produce a uniform analysis, we adopted the spectroscopic constraints from Bruntt et al. (2012), limiting the available sample to 46 stars. Four of these targets are evolved subgiants with too many mixed modes for successful automated modeling, so our final sample includes 42 stars. Following Chaplin et al. (2014), we adopted larger uncertainties on $T_{\text {eff }}( \pm 84$ $\mathrm{K}$; see Figure 1) that fold in a systematic error of $59 \mathrm{~K}$, as suggested by Torres et al. (2012). For 12 stars with Hipparcos parallaxes (van Leeuwen 2007), we used the spectroscopic $T_{\text {eff }}$ to obtain bolometric corrections from Flower (1996). Following Torres (2010), we adopted $M_{\mathrm{bol}, \odot}=4.73 \pm 0.03$ and used the extinction estimates from Ammons et al. (2006) to derive luminosity constraints.

\section{STELLAR MODELING APPROACH}

The AMP (Woitaszek et al. 2009) is a web-based interface to the stellar model-fitting pipeline described in detail by Metcalfe et al. (2009). The underlying science code uses a parallel genetic algorithm (GA; Metcalfe \& Charbonneau 2003) on XSEDE supercomputing resources to optimize the match between asteroseismic models produced by the Aarhus stellar evolution code (ASTEC; Christensen-Dalsgaard 2008a) and adiabatic pulsation code (ADIPLS; Christensen-Dalsgaard 2008 b) and a given set of observational constraints. Mathur et al. (2012) were the first to apply AMP to a large sample of Kepler targets, motivating several improvements to the physical inputs and fitting procedures that are described below.

\subsection{Updated Physics}

The version of AMP that was used for the models presented by Mathur et al. (2012) was configured to use the Grevesse \& Noels (1993) solar mixture with the OPAL 2005 equation of state (see Rogers \& Nayfonov 2002) and the most recent OPAL opacities (see Iglesias \& Rogers 1996), supplemented by Alexander \& Ferguson (1994) opacities at low temperatures. The updated version of AMP uses the low-T opacities from Ferguson et al. (2005). We have also updated the default nuclear reaction rates, replacing the Bahcall \& Pinsonneault (1995) rates with those from the NACRE collaboration (Angulo et al. 1999). Convection is still described by the mixing-length theory from Böhm-Vitense (1958) without overshoot, and we continue to include the effects of helium diffusion and settling as described by Michaud \& Proffitt (1993). To correct the model frequencies for so-called "surface effects" due to incomplete modeling of the near-surface layers, we use the empirical prescription of Kjeldsen et al. (2008).

We originally performed our analysis shortly after the publication of asteroseismic constraints by Appourchaux et al. (2012), using the updated physics described above but without modifying the fitting procedures. The approach used by Mathur et al. (2012) simultaneously optimized the match between the models and two sets of constraints: (1) the individual oscillation frequencies and (2) the atmospheric parameters from spectroscopy. This procedure generally yielded stellar radii, masses, and ages that were consistent with empirical scaling relations and grid-based modeling of the global oscillation properties $\left(\Delta v\right.$ and $\left.v_{\max }\right)$-but with significantly improved precision. However, the optimal models for the 22 targets included six stars with an initial helium mass fraction $Y_{\mathrm{i}}$ significantly below the primordial value from standard Big Bang nucleosynthesis $\left(Y_{\mathrm{P}}=0.2482 \pm 0.0007\right.$; Steigman 2010), and four additional stars that were marginally below $Y_{\mathrm{P}}$. The original motivation for including these sub-primordial values in the search was a recognition that there could be systematic errors in the determination of $Y_{\mathrm{i}}$, but the source of the potential bias was not identified. Our first attempts to fit the data described in Section 2 using the same methods as Mathur et al. (2012) were plagued by an even higher fraction of models with low initial helium, so we revised our procedures.

\subsection{Updated Fitting Procedures}

As part of a study of convective cores in two Kepler targets, AMP was compared to several other fitting methods by Silva Aguirre et al. (2013). In addition to the individual frequencies and spectroscopic constraints, some of these methods also used sets of frequency ratios that eliminated the need to 
correct the model frequencies for surface effects (Roxburgh $\&$ Vorontsov 2003). A comparison of the AMP results with models that used the frequency ratios as additional constraints revealed systematic differences in the interior structure that were correlated with the initial helium abundance. We subsequently modified the AMP optimization procedure to try to avoid this bias, by adopting the frequency ratios as additional constraints and by reducing the weight at higher frequency, where the surface correction is larger (see details below).

The ratios proposed by Roxburgh \& Vorontsov (2003) are constructed from individual frequency separations, including the large separations defined by $\Delta v_{\ell}(n)=v_{n, \ell}-v_{n-1, \ell}$, and the small separations defined by $d_{\ell, \ell+2}(n)=v_{n, \ell}-v_{n-1, \ell+2}$. These can be used to define one set of ratios that relates the small separation between modes of degree 0 and 2 to the large separation of $\ell=1$ modes at the same radial order:

$$
r_{02}(n)=\frac{d_{0,2}(n)}{\Delta v_{1}(n)} .
$$

Note that these ratios involve modes with all three degrees. Another set of ratios only involves the small and large separations between modes of degree 0 and 1 :

$$
r_{01}(n)=\frac{d_{01}(n)}{\Delta v_{1}(n)}, \quad r_{10}(n)=\frac{d_{10}(n)}{\Delta v_{0}(n+1)},
$$

where $d_{01}(n)$ and $d_{10}(n)$ are five frequency smoothed small separations defined by Equations (4) and (5) in Roxburgh \& Vorontsov (2003). This smoothing introduces correlations between the individual ratios that more than double the effective uncertainties, ${ }^{33}$ but it also shifts some weight from the center of the frequency range toward the edges where the $\mathrm{S} / \mathrm{N}$ of the modes is lower. ${ }^{34}$ To account for these correlations without shifting weight toward the edges of the frequency range, we adopted $3 \sigma$ uncorrelated uncertainties on all ratios from Equation (2). In addition, Silva Aguirre et al. (2013) noted that the ratios formed from the highest radial orders were typically unreliable due to large line widths, and recommended that they be excluded from the set of constraints. We excluded all ratios that are centered on frequencies from the highest three radial orders. Hereafter, we refer to the set of ratios $r_{02}(n)$ as $r_{02}$ and the set of ratios $r_{01}(n)$ and $r_{10}(n)$ as $r_{010}$.

Although the frequency ratios help to discriminate between families of models that provide comparable matches to the other sets of constraints, the individual frequencies contain additional information that we would like to exploit. The primary difficulty is that the model frequencies need to be corrected for surface effects, and the commonly used empirical correction (Kjeldsen et al. 2008) appears to inject a bias in the determination of some stellar properties. What is the source of this bias, and how can we mitigate it? Essentially, Kjeldsen et al. assumed that the differences between the observed and optimal model frequencies can be described by

$$
v_{\mathrm{obs}}-v_{\mathrm{mod}} \approx a_{0}\left(\frac{v}{v_{0}}\right)^{b},
$$

\footnotetext{
33 We examined a specific case from Silva Aguirre et al. (2013) and compared the quadratic sum of all terms in the covariance matrix to the diagonal element for each ratio. We determined that the off-diagonal elements inflate the effective uncertainty by a factor of two to four with the largest boost near the center of the observed frequency range.

34 Ratios near the edges of the observed frequency range are directly correlated with fewer than four other ratios, so the correlated errors are not inflated as much relative to the diagonal elements of the covariance matrix and these less certain ratios are assigned higher relative weights.
}

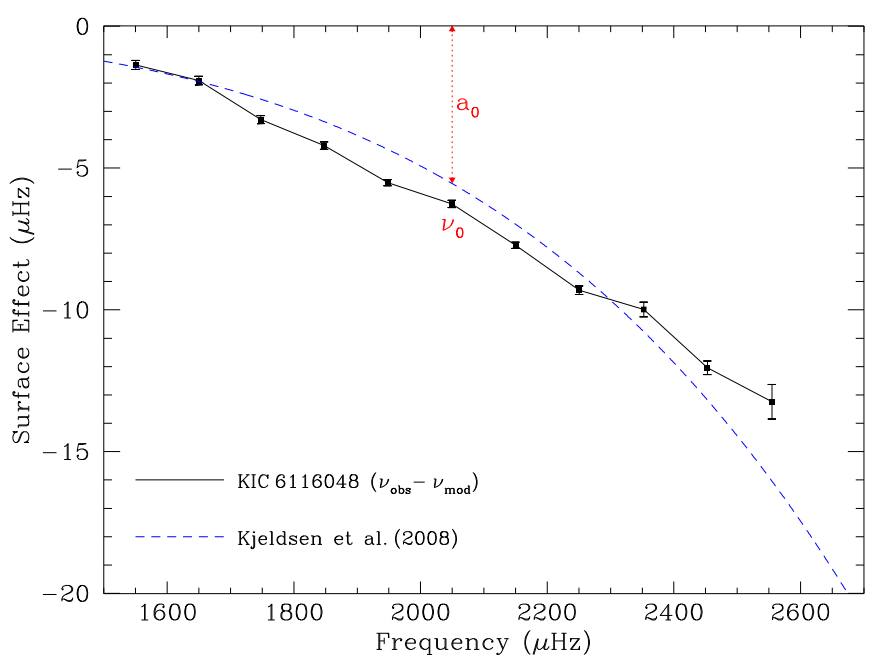

Figure 2. Comparison of the actual surface effect and the empirical correction of Kjeldsen et al. (2008) for the AMP model of KIC 6116048. Differences between the observed $\ell=0$ frequencies and those of the AMP model (connected points) are reasonably well represented by the empirical correction (dashed line) with amplitude $a_{0}$ at the reference frequency $v_{0}$, but it substantially overestimates the correction at high frequencies.

(A color version of this figure is available in the online journal.)

where $a_{0}$ is the size of the correction at a reference frequency $v_{0}$ (typically chosen to be $v_{\max }$ ), and the exponent $b$ is fixed at a solar-calibrated value near 4.9. They demonstrated that this simple parameterization ${ }^{35}$ can adequately describe the frequency differences between the observations and models of several solar-type stars, including $\beta$ Hyi and $\alpha$ Cen A and B. They cautioned that the value of the exponent depends on the number of radial orders considered for the solar calibration, varying from 4.4-5.25 when including 7-13 orders. To facilitate comparisons with previous work, we adopted the solar-calibrated value $b=4.82$ determined by Metcalfe et al. (2009). For mixed modes we scaled the surface correction by the mode inertia ratio, as described in Brandão et al. (2011).

The actual solar surface effect appears more linear at high frequencies (Christensen-Dalsgaard et al. 1996), so assuming any fixed exponent will tend to over-correct the highest-order modes (see Figure 2). This tendency appears to interact with intrinsic parameter correlations - in particular, the well-known correlation between mass and initial helium abundance in stellar models - to favor higher-mass low-helium models that fit the frequencies better while getting the interior structure wrong. Including the frequency ratios as constraints favors the lower-mass higher-helium models, but it does not eliminate the bias caused by the high-frequency modes. To mitigate this bias, we adopted an uncertainty for each frequency that is the quadratic sum of the statistical error and half the surface correction (Bevington \& Robinson 1992). In doing so, we are acknowledging that surface effects represent a systematic error in the models (Guenther \& Brown 2004). We also imposed a penalty on models with $Y_{\mathrm{i}}<Y_{\mathrm{P}}$, such that the spectroscopic quality metric (see Section 3.3) was inflated by 1 for every 0.01 that $Y_{\mathrm{i}}$ fell below $Y_{\mathrm{P}}$. Although this does not explicitly rule out low-helium solutions, it does require

\footnotetext{
35 Note that Kjeldsen et al. (2008) also scaled the model frequencies by a homology factor $r$ to provide a better match to the observations. By definition, the best model should have $r=1$. The net effect of applying homology scaling to every model sampled by the GA is to decrease the dynamic range of the frequency $\chi^{2}$ space, so we omitted this term from our surface correction.
} 

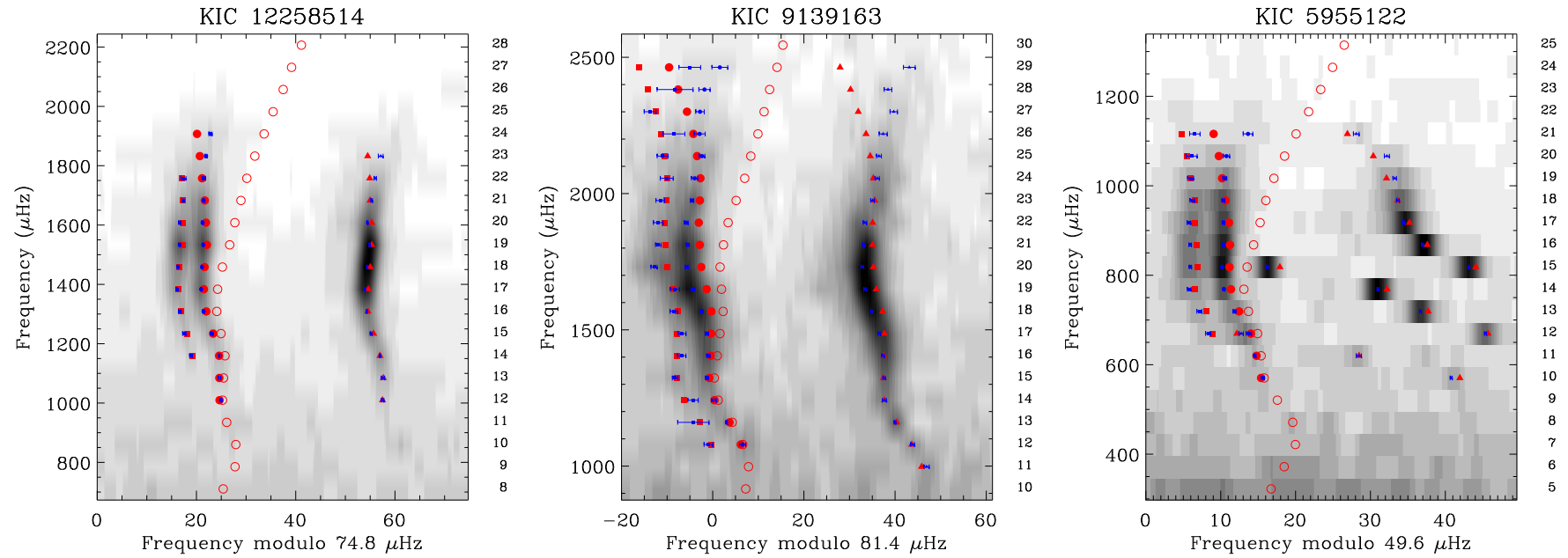

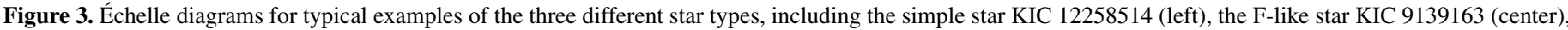

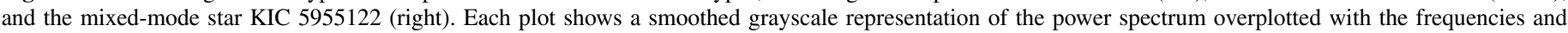

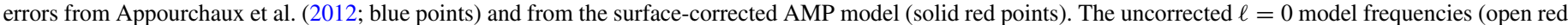
circles) are shown to illustrate the size of the surface effect, and the $\ell=0$ radial order is given on the right axis.

(An extended, color version of this figure is available in the online journal.)

that they provide a substantially better match to the other sets of constraints to be considered superior. Without a precise constraint on the luminosity and/or radius, this approach is required even to recover accurate solar properties from Sun-asa-star helioseismic data (Metcalfe et al. 2009).

\subsection{Customization by Star Type}

Appourchaux et al. (2012) categorized their sample of 61 asteroseismic targets into three classes, based on the appearance of the oscillation modes in an échelle diagram (Grec et al. 1980). Dividing the frequency spectrum into segments having width equal to the large separation and then stacking them vertically, modes with the same spherical degree form approximately vertical ridges for simple stars like the Sun (see Figure 3). Significantly hotter main-sequence stars have larger intrinsic line widths, blurring the individual modes and complicating the identification of mode geometry (F-like stars). Finally, the $\ell=1$ ridge in subgiants can be disrupted when buoyancy modes in the evolved stellar core couple with pressure modes in the envelope, creating an avoided crossing (Osaki 1975; Aizenman et al. 1977) that leads to deviations from the regular frequency spacing (mixed-mode stars). This final category is usually unambiguous, but there is no clear dividing line between the first two.

Appourchaux et al. (2012) suggested a boundary between the simple and F-like stars at an effective temperature near $6400 \mathrm{~K}$ or a line width at maximum mode height around $4 \mu \mathrm{Hz}$ (see White et al. 2012). We adopted a slightly different convention based on whether or not the $\ell=0$ and $\ell=2$ ridges in the échelle diagram are cleanly separated. This led us to treat 5 stars as F-like that were identified as simple by Appourchaux et al.: KIC 3632418, 7206837, 8228742, 9139163, and 10162436. In addition, KIC 3424541 (originally classified as F-like) and KIC 10018963 (classified as simple by Appourchaux et al.) both show evidence of avoided crossings, so we treated them as mixed-mode stars.

The use of frequency ratios as additional constraints for asteroseismic model-fitting can improve the uniqueness of the solution, but the ratios cannot all be used for certain types of stars. For simple stars, the large and small spacings can be measured cleanly, and the underlying assumption that the frequencies are all pure $p$-modes is justified. In this case, AMP attempts to match four sets of observational constraints simultaneously: (1) the individual oscillation frequencies, with uncertainties inflated in proportion to the surface correction, (2) the ratios $r_{010}$ with $3 \sigma$ uncorrelated statistical uncertainties, (3) the ratios $r_{02}$ with errors propagated from the quoted frequency uncertainties, and (4) the spectroscopic and other constraints, such as a luminosity or interferometric radius. A normalized $\chi^{2}$ is calculated for each of these sets of constraints:

$$
\chi^{2}=\frac{1}{N} \sum_{i=1}^{N}\left(\frac{O_{i}-C_{i}}{\sigma_{i}}\right)^{2}
$$

where $O_{i}$ and $C_{i}$ are the sets of $N$ observed and calculated quantities, and $\sigma_{i}$ are the associated uncertainties. ${ }^{36}$ The GA then attempts to minimize the average of the $\chi^{2}$ values for the adopted sets of constraints. ${ }^{37}$ This approach recognizes that each oscillation frequency is not completely independent, but it uses the information content in several different ways to create metrics that can be traded off against each other and/or against the spectroscopic constraints.

These procedures must be modified slightly for F-like and mixed-mode stars. In F-like stars, large line widths make the measurement of $\ell=0$ and $\ell=2$ frequencies more difficult. Consequently, the small spacings $d_{0,2}(n)$ are compromised and the ratios $r_{02}$ are unreliable. In this case, set (3) above is excluded from consideration, and the GA uses the average $\chi^{2}$ for the remaining three sets of constraints. For mixed-mode stars, the $\ell=1$ frequencies are not pure $p$-modes, so the theoretical insensitivity of the ratios $r_{010}$ to the near-surface layers is no longer valid and they lose their utility. This is not limited to the modes that are immediately adjacent to an avoided crossing - several radial orders on either side are generally perturbed, depending on the strength of the mode

\footnotetext{
36 We did not consider covariances for any of our quality metrics. Lebreton \& Goupil (2014) recently demonstrated that including a full treatment of correlations has a negligible impact on the derived stellar properties.

37 This decision compromises the statistical purity of our metric, as discussed in Section 4.1, so future updates to AMP will preserve the individual $\chi^{2}$ values for subsequent error analysis.
} 
Table 1

Properties of the Optimal Models and Surface Correction from AMP

\begin{tabular}{|c|c|c|c|c|c|c|c|c|c|}
\hline KIC & $R / R_{\odot}^{\mathrm{a}}$ & $M / M_{\odot}^{\mathrm{a}}$ & $t / \mathrm{Gyr}^{\mathrm{a}}$ & $Z$ & $Y_{\mathrm{i}}$ & $\alpha$ & $a_{0}$ & $f$ & $\mathrm{AMP}^{\mathrm{b}}$ \\
\hline \multicolumn{10}{|l|}{ Simple stars } \\
\hline 3427720 & $1.125 \pm 0.014$ & $1.13 \pm 0.04$ & $2.23 \pm 0.17$ & $0.0168 \pm 0.0016$ & $0.248 \pm 0.020$ & $1.98 \pm 0.11$ & -3.65 & 1.2 & 641 \\
\hline 6116048 & $1.219 \pm 0.009$ & $1.01 \pm 0.03$ & $6.23 \pm 0.37$ & $0.0118 \pm 0.0011$ & $0.255 \pm 0.014$ & $1.80 \pm 0.10$ & -5.55 & 6.2 & 642 \\
\hline 6603624 & $1.181 \pm 0.015$ & $1.09 \pm 0.03$ & $8.11 \pm 0.46$ & $0.0309 \pm 0.0029$ & $0.250 \pm 0.021$ & $2.12 \pm 0.09$ & -1.65 & 10.3 & 643 \\
\hline 6933899 & $1.599 \pm 0.018$ & $1.14 \pm 0.03$ & $6.87 \pm 0.34$ & $0.0203 \pm 0.0022$ & $0.257 \pm 0.014$ & $2.10 \pm 0.07$ & -1.98 & 2.8 & 497 \\
\hline 7871531 & $0.874 \pm 0.008$ & $0.84 \pm 0.02$ & $9.15 \pm 0.47$ & $0.0125 \pm 0.0014$ & $0.263 \pm 0.018$ & $2.02 \pm 0.12$ & -4.07 & 4.2 & 523 \\
\hline 8006161 & $0.947 \pm 0.007$ & $1.04 \pm 0.02$ & $5.04 \pm 0.17$ & $0.0427 \pm 0.0052$ & $0.259 \pm 0.015$ & $2.22 \pm 0.08$ & -1.99 & 2.0 & 494 \\
\hline 8394589 & $1.116 \pm 0.019$ & $0.94 \pm 0.04$ & $2.92 \pm 0.18$ & $0.0082 \pm 0.0005$ & $0.308 \pm 0.028$ & $1.62 \pm 0.07$ & -9.68 & 1.5 & 526 \\
\hline 8694723 & $1.436 \pm 0.024$ & $0.96 \pm 0.03$ & $4.90 \pm 0.54$ & $0.0058 \pm 0.0006$ & $0.298 \pm 0.025$ & $1.52 \pm 0.10$ & -7.70 & 36.8 & 516 \\
\hline 8760414 & $1.010 \pm 0.004$ & $0.78 \pm 0.01$ & $3.69 \pm 0.74$ & $0.0032 \pm 0.0003$ & $0.238 \pm 0.006$ & $1.90 \pm 0.12$ & -6.01 & 20.3 & 644 \\
\hline 9098294 & $1.154 \pm 0.009$ & $1.00 \pm 0.03$ & $7.28 \pm 0.51$ & $0.0143 \pm 0.0018$ & $0.252 \pm 0.017$ & $2.00 \pm 0.12$ & -4.76 & 1.6 & 509 \\
\hline 9139151 & $1.146 \pm 0.011$ & $1.14 \pm 0.03$ & $1.71 \pm 0.19$ & $0.0224 \pm 0.0014$ & $0.289 \pm 0.018$ & $2.04 \pm 0.07$ & -4.67 & 0.7 & 508 \\
\hline 9955598 & $0.883 \pm 0.008$ & $0.89 \pm 0.02$ & $6.72 \pm 0.20$ & $0.0231 \pm 0.0017$ & $0.291 \pm 0.017$ & $2.06 \pm 0.09$ & -2.69 & 3.6 & 524 \\
\hline 10454113 & $1.250 \pm 0.015$ & $1.19 \pm 0.04$ & $2.03 \pm 0.29$ & $0.0168 \pm 0.0012$ & $0.252 \pm 0.018$ & $1.86 \pm 0.08$ & -4.67 & 2.6 & 645 \\
\hline 10644253 & $1.108 \pm 0.016$ & $1.13 \pm 0.05$ & $1.07 \pm 0.25$ & $0.0239 \pm 0.0024$ & $0.290 \pm 0.025$ & $1.96 \pm 0.12$ & -7.48 & 1.5 & 527 \\
\hline 10963065 & $1.213 \pm 0.008$ & $1.05 \pm 0.02$ & $4.30 \pm 0.23$ & $0.0118 \pm 0.0010$ & $0.262 \pm 0.012$ & $1.84 \pm 0.08$ & -5.34 & 8.4 & 518 \\
\hline 11244118 & $1.589 \pm 0.026$ & $1.10 \pm 0.05$ & $6.43 \pm 0.58$ & $0.0272 \pm 0.0034$ & $0.310 \pm 0.031$ & $2.16 \pm 0.19$ & -2.08 & 10.0 & 499 \\
\hline 12009504 & $1.375 \pm 0.015$ & $1.12 \pm 0.03$ & $3.64 \pm 0.26$ & $0.0152 \pm 0.0011$ & $0.282 \pm 0.023$ & $1.76 \pm 0.06$ & -5.80 & 7.0 & 498 \\
\hline 12258514 & $1.573 \pm 0.010$ & $1.19 \pm 0.03$ & $4.03 \pm 0.32$ & $0.0197 \pm 0.0015$ & $0.290 \pm 0.017$ & $1.88 \pm 0.08$ & -3.57 & 3.9 & 490 \\
\hline \multicolumn{10}{|l|}{ F-like stars } \\
\hline 1435467 & $1.641 \pm 0.027$ & $1.27 \pm 0.05$ & $1.87 \pm 0.14$ & $0.0203 \pm 0.0016$ & $0.317 \pm 0.023$ & $1.74 \pm 0.05$ & -5.41 & 8.9 & 552 \\
\hline 2837475 & $1.592 \pm 0.027$ & $1.39 \pm 0.06$ & $0.83 \pm 0.12$ & $0.0224 \pm 0.0018$ & $0.315 \pm 0.029$ & $2.12 \pm 0.12$ & -4.08 & 1.9 & 553 \\
\hline 3632418 & $1.835 \pm 0.034$ & $1.27 \pm 0.03$ & $2.88 \pm 0.38$ & $0.0143 \pm 0.0015$ & $0.288 \pm 0.027$ & $1.88 \pm 0.08$ & -3.51 & 14.2 & 646 \\
\hline 3733735 & $1.367 \pm 0.023$ & $1.32 \pm 0.04$ & $0.12 \pm 0.06$ & $0.0179 \pm 0.0011$ & $0.300 \pm 0.021$ & $1.72 \pm 0.11$ & -5.33 & 1.4 & 554 \\
\hline 6508366 & $2.081 \pm 0.021$ & $1.36 \pm 0.04$ & $2.25 \pm 0.15$ & $0.0168 \pm 0.0011$ & $0.305 \pm 0.022$ & $1.84 \pm 0.09$ & -2.77 & 2.9 & 536 \\
\hline 6679371 & $2.186 \pm 0.015$ & $1.56 \pm 0.03$ & $1.81 \pm 0.12$ & $0.0191 \pm 0.0019$ & $0.245 \pm 0.014$ & $1.56 \pm 0.09$ & -2.70 & 6.4 & 647 \\
\hline 7103006 & $1.898 \pm 0.026$ & $1.43 \pm 0.05$ & $1.49 \pm 0.14$ & $0.0247 \pm 0.0025$ & $0.313 \pm 0.026$ & $1.88 \pm 0.10$ & -3.78 & 12.1 & 530 \\
\hline 7206837 & $1.555 \pm 0.016$ & $1.46 \pm 0.05$ & $0.22 \pm 0.04$ & $0.0299 \pm 0.0012$ & $0.271 \pm 0.022$ & $1.34 \pm 0.08$ & -10.35 & 13.1 & 556 \\
\hline 8228742 & $1.809 \pm 0.014$ & $1.27 \pm 0.02$ & $3.84 \pm 0.29$ & $0.0147 \pm 0.0015$ & $0.247 \pm 0.017$ & $1.64 \pm 0.06$ & -5.02 & 24.9 & 648 \\
\hline 9139163 & $1.532 \pm 0.021$ & $1.36 \pm 0.03$ & $1.07 \pm 0.16$ & $0.0272 \pm 0.0014$ & $0.302 \pm 0.017$ & $1.84 \pm 0.07$ & -4.33 & 7.3 & 557 \\
\hline 9206432 & $1.479 \pm 0.014$ & $1.40 \pm 0.03$ & $0.19 \pm 0.07$ & $0.0290 \pm 0.0015$ & $0.312 \pm 0.011$ & $1.68 \pm 0.10$ & -5.94 & 5.5 & 532 \\
\hline 9812850 & $1.745 \pm 0.024$ & $1.39 \pm 0.05$ & $1.68 \pm 0.13$ & $0.0162 \pm 0.0011$ & $0.252 \pm 0.022$ & $1.26 \pm 0.09$ & -7.82 & 6.1 & 649 \\
\hline 10162436 & $1.903 \pm 0.020$ & $1.23 \pm 0.02$ & $2.86 \pm 0.33$ & $0.0162 \pm 0.0009$ & $0.316 \pm 0.012$ & $1.58 \pm 0.08$ & -5.46 & 24.2 & 539 \\
\hline 10355856 & $1.667 \pm 0.015$ & $1.32 \pm 0.03$ & $1.54 \pm 0.13$ & $0.0152 \pm 0.0009$ & $0.280 \pm 0.018$ & $1.18 \pm 0.15$ & -6.84 & 7.1 & 650 \\
\hline 11081729 & $1.382 \pm 0.021$ & $1.26 \pm 0.03$ & $0.86 \pm 0.21$ & $0.0162 \pm 0.0006$ & $0.303 \pm 0.017$ & $2.00 \pm 0.11$ & -6.72 & 7.1 & 551 \\
\hline 11253226 & $1.551 \pm 0.019$ & $1.41 \pm 0.05$ & $0.56 \pm 0.20$ & $0.0197 \pm 0.0012$ & $0.279 \pm 0.025$ & $1.22 \pm 0.26$ & -7.64 & 4.7 & 555 \\
\hline \multicolumn{10}{|c|}{ Mixed-mode stars } \\
\hline 3424541 & $2.526 \pm 0.065$ & $1.64 \pm 0.04$ & $2.28 \pm 0.13$ & $0.0309 \pm 0.0036$ & $0.257 \pm 0.018$ & $1.74 \pm 0.12$ & -5.44 & 2.3 & 636 \\
\hline 5955122 & $2.042 \pm 0.025$ & $1.12 \pm 0.05$ & $5.26 \pm 0.58$ & $0.0143 \pm 0.0023$ & $0.290 \pm 0.022$ & $1.76 \pm 0.15$ & -2.69 & 15.6 & 537 \\
\hline 7747078 & $1.889 \pm 0.023$ & $1.06 \pm 0.05$ & $6.26 \pm 0.92$ & $0.0103 \pm 0.0019$ & $0.271 \pm 0.020$ & $1.76 \pm 0.20$ & -5.63 & 38.6 & 541 \\
\hline 7976303 & $1.961 \pm 0.041$ & $1.10 \pm 0.05$ & $4.78 \pm 0.58$ & $0.0077 \pm 0.0015$ & $0.268 \pm 0.016$ & $1.92 \pm 0.19$ & -7.36 & 150.0 & 538 \\
\hline 8026226 & $2.753 \pm 0.041$ & $1.50 \pm 0.03$ & $2.23 \pm 0.13$ & $0.0125 \pm 0.0010$ & $0.247 \pm 0.007$ & $1.28 \pm 0.13$ & -4.99 & 32.5 & 651 \\
\hline 8524425 & $1.733 \pm 0.015$ & $1.00 \pm 0.07$ & $7.98 \pm 0.46$ & $0.0197 \pm 0.0022$ & $0.313 \pm 0.041$ & $1.72 \pm 0.11$ & -3.27 & 15.2 & 564 \\
\hline 10018963 & $1.915 \pm 0.020$ & $1.18 \pm 0.03$ & $4.36 \pm 0.34$ & $0.0097 \pm 0.0011$ & $0.255 \pm 0.010$ & $1.96 \pm 0.11$ & -2.66 & 113.2 & 544 \\
\hline 11026764 & $2.106 \pm 0.025$ & $1.27 \pm 0.06$ & $5.00 \pm 0.53$ & $0.0197 \pm 0.0029$ & $0.254 \pm 0.016$ & $2.10 \pm 0.37$ & -1.62 & 4.6 & 567 \\
\hline
\end{tabular}

Notes.

${ }^{\text {a }}$ Formal uncertainties do not include a typical systematic component of $1.3 \%$ for radius, $3.7 \%$ for mass, and $12 \%$ for age.

b Comprehensive model output is available at http://amp.phys.au.dk/browse/simulation/\#\#\#.

coupling (Deheuvels \& Michel 2011; Benomar et al. 2013). In addition, note that the ratios $r_{02}$ in Equation (1) depend on the large separations $\Delta v_{1}(n)$, which are also contaminated by mixed modes. So in this case we must exclude both sets (2) and (3) above, leaving only the individual frequencies (assigned double weight ${ }^{38}$ ) and spectroscopic constraints. The modified treatment of errors on the individual frequencies is still in effect for the

\footnotetext{
38 For mixed-mode stars, the information content of the individual frequencies is not redistributed among any sets of frequency ratios, so we compensate by assigning double weight to the frequencies relative to the spectroscopic constraints.
}

mixed-mode stars, so the new procedure is different from the approach taken by Mathur et al. (2012).

\section{RESULTS}

The properties derived by AMP for our sample of 42 mainsequence and subgiant Kepler targets are listed in Table 1. For each star in the three categories, we give the Kepler Input Catalog (KIC; Brown et al. 2011) number, the asteroseismic radius $R$, mass $M$, age $t$, heavy element mass fraction $Z$, initial helium mass fraction $Y_{\mathrm{i}}$, mixing-length $\alpha$, and amplitude of the surface correction $a_{0}$ at the reference frequency $v_{\max }$ (where 
$v_{\max }$ was taken from Appourchaux et al. 2012). As an indication of the relative quality of each model, we list an average of the normalized $\chi^{2}$ values discussed in Section 3.3- similar to the metric that was used by the GA to identify the optimal model, but using the original frequency errors from Appourchaux et al. (2012) rather than inflating them in proportion to the surface correction. This modified metric $f$ is more useful for comparing the quality of the models for different stars, and can be used in conjunction with the échelle diagrams in Figure 3 to judge the reliability of each result. Some of the models are clearly better representations of the observations than others (see discussion below), so we caution readers not to treat them all as equivalent. In the final column of Table 1, we list the AMP run number so that interested readers can access a comprehensive archive with the evolution and structure of each stellar model.

Figure 3 shows échelle diagrams for typical examples of each of the three different star types, with similar plots for all 42 stars included in the online version of the Journal. A common feature in many of these diagrams is a divergence between the observations (blue points) and the surface-corrected model frequencies (solid red points), which appear to curve off toward the left at higher frequencies. This feature is most obvious in the F-like star shown in the center panel, which has a larger number of observed radial orders. As discussed in Section 3.2, this is an indication that the empirical surface correction of Kjeldsen et al. (2008) tends to over-correct the highest order modes, motivating our decision to decrease the weight of these frequencies in proportion to the size of the surface correction (i.e., the difference between the solid and open red circles in each plot). Thus, the primary constraints from the individual frequencies are concentrated at low frequency, while the ratios capture the information content of the higher frequencies (excluding the highest three radial orders; see Section 3.2). Forcing a better fit to the high frequency modes, under the current approach to the surface correction, leads to the systematic bias toward low-helium solutions that affected the results of Mathur et al. (2012).

\subsection{Statistical and Systematic Uncertainties}

The assessment of uncertainties on the adjustable parameters and other model properties requires some degree of pragmatism. Our previous approach, based on the local shape of the $\chi^{2}$ surface using singular value decomposition (SVD; Creevey 2009; Metcalfe et al. 2009), generally fails to capture uncertainties due to non-uniqueness of the solution-a common outcome when we combine several different metrics of model quality. Rather than accept the underestimated uncertainties from SVD, we opted for a more conservative approach using an ensemble of the best models sampled by the GA on its way to finding the optimal solution. First, we ranked the 50,000-80,000 unique models for each star by the average $\chi^{2}$ value and assigned each model a likelihood:

$$
\mathcal{L}=\exp \left(-\chi^{2} / 2\right)
$$

Next, we calculated a likelihood-weighted mean value and standard deviation for each parameter, including additional models in the mean until the uncertainty on $[Z / X]_{i}$ was comparable to the observational error on $[\mathrm{M} / \mathrm{H}]$. This is a first approximation, since helium diffusion and settling gradually changes the value of $X$ as the model evolves to its final age. Using these uncertainties for the five adjustable model parameters $\left(M, t, Z, Y_{\mathrm{i}}, \alpha\right)$, we rescaled the covariance matrix from the optimal solution and calculated a set of models to define the $1 \sigma$ error ellipse. The uncertainties on other model properties, such as $R$ and $T_{\text {eff }}$, were determined from the range of values represented in these $1 \sigma$ models. Finally, we refined the number of models that were included in the mean so that the output uncertainty on the model $T_{\text {eff }}$ was equal to the input error on the spectroscopic $T_{\text {eff }}$. This procedure leads to an inherently conservative estimate of the uncertainties, because it implicitly assumes that the asteroseismic data do not contribute to the determination of $T_{\text {eff }}$ in the final solution. With the ensemble of best models defined in this way for each star, we used them to determine the likelihood-weighted standard deviation (taken to be the uncertainty) on each parameter of the best solution identified by the GA (for a detailed example, see the Appendix).

It is important to emphasize that the resulting uncertainties, listed in Table 1, do not include systematic errors from our particular choice of model physics or fitting strategy. Chaplin et al. (2014) used results from six pipeline methodologies coupled to 11 different model grids to quantify the associated systematic uncertainties. They found that, in addition to the statistical uncertainties from a given model grid and fitting methodology, the systematic errors for stars with a spectroscopic $T_{\text {eff }}$ and $[\mathrm{M} / \mathrm{H}]$ were typically $1.3 \%$ on the radius, $3.7 \%$ on the mass, and $12 \%$ on the age. Although these additional systematic errors may have an impact on the absolute values of the stellar properties derived by AMP, the uniformity of the data sources and modeling approach means that the relative values can be considered as reliable as the uncertainties listed in Table 1. Readers who wish to combine our results with those from other sources should consider the additional systematic errors noted above.

\subsection{Comparison with Other Methods}

Setting aside the question of absolute accuracy for the derived stellar properties, we can compare the internal precision of the AMP results to other common methods of inferring asteroseismic radii, masses and ages. Such a comparison can quantify the benefits of modeling the individual frequencies, relative to using only the global oscillation properties such as $\Delta v$ and $v_{\max }$. Empirical scaling relations can provide modelindependent estimates of the asteroseismic radius and mass using only the observed values of $\Delta v, v_{\max }$ and $T_{\text {eff }}$ (Kjeldsen $\&$ Bedding 1995). In Figure 4, we compare the radii (left) and masses (right) derived from the scaling relations (SR) to those found by AMP. The top panels compare the actual values and associated uncertainties, while the bottom panels show differences between the estimates normalized by the uncertainty on the difference. For clarity, simple (black circles), F-like (red diamonds), and mixed-mode stars (blue squares) are shown in different colors. The median uncertainties from the scaling relations are $4.2 \%$ on the radius and $6.8 \%$ on the mass. The scaling relation values for F-like stars are systematically higher on average than the estimates from AMP, but the agreement is generally better than $2 \sigma$.

In Figure 5, we show a similar comparison of AMP results with grid-modeling from Chaplin et al. (2014), including the asteroseismic radii (left), masses (center), and ages (right). These grid-modeling (GM) results use the same spectroscopic constraints from Bruntt et al. (2012), but the adopted model grid (yielding values closest to the median over all grids and methods) came from the GARSTEC code (Weiss \& Schlattl 2008). The median uncertainties from grid-modeling of this sample are $2.1 \%$ on the radius, $5.5 \%$ on the mass, and $20 \%$ 

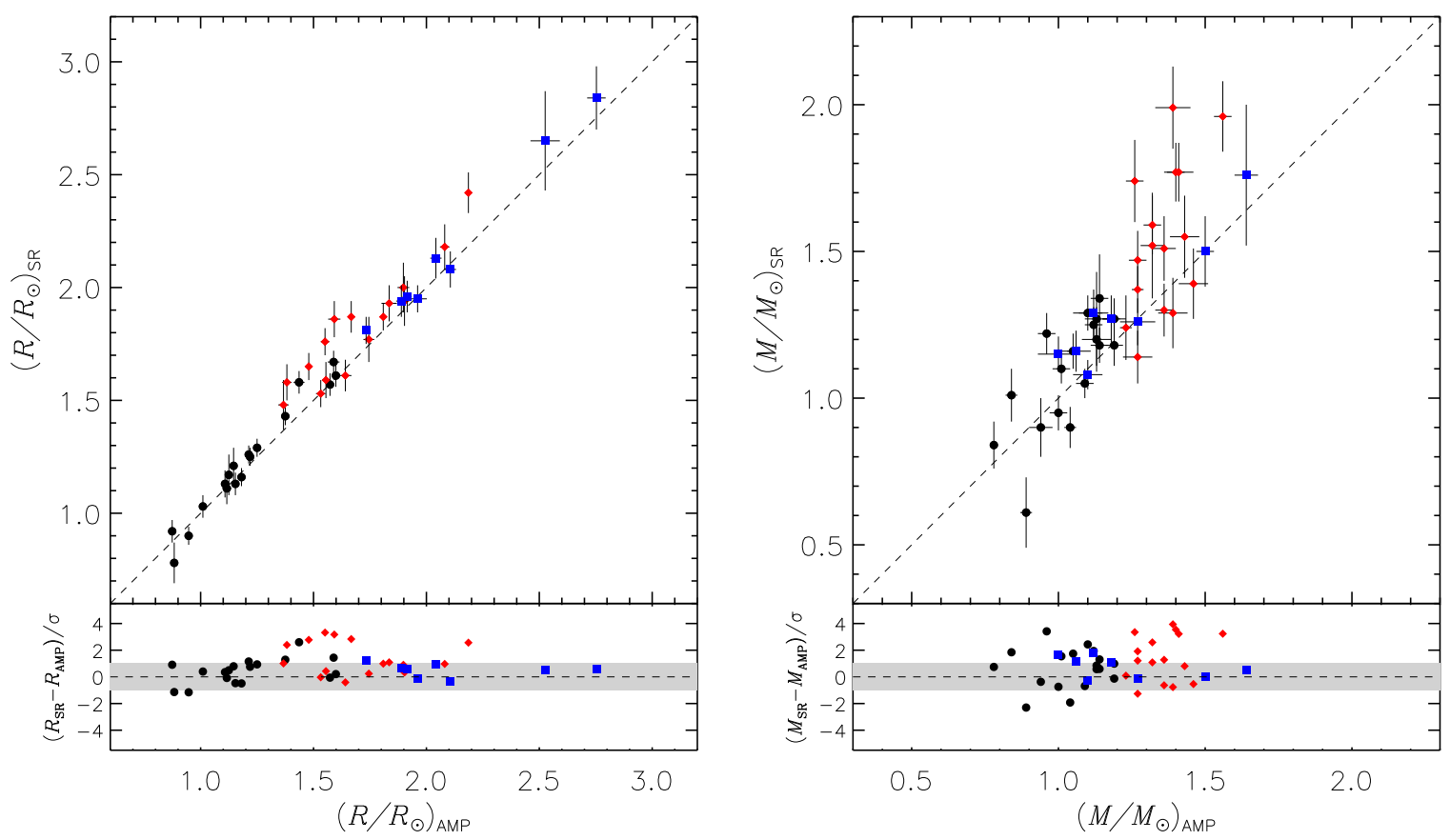

Figure 4. Comparison of the asteroseismic radii (left) and masses (right) derived from scaling relations (SR) with the AMP estimates from Table 1, including the simple (black circles), F-like (red diamonds), and mixed-mode stars (blue squares). The top panels compare the values and uncertainties, while the bottom panels show the differences between the estimates normalized by the uncertainty on the difference.

(A color version of this figure is available in the online journal.)
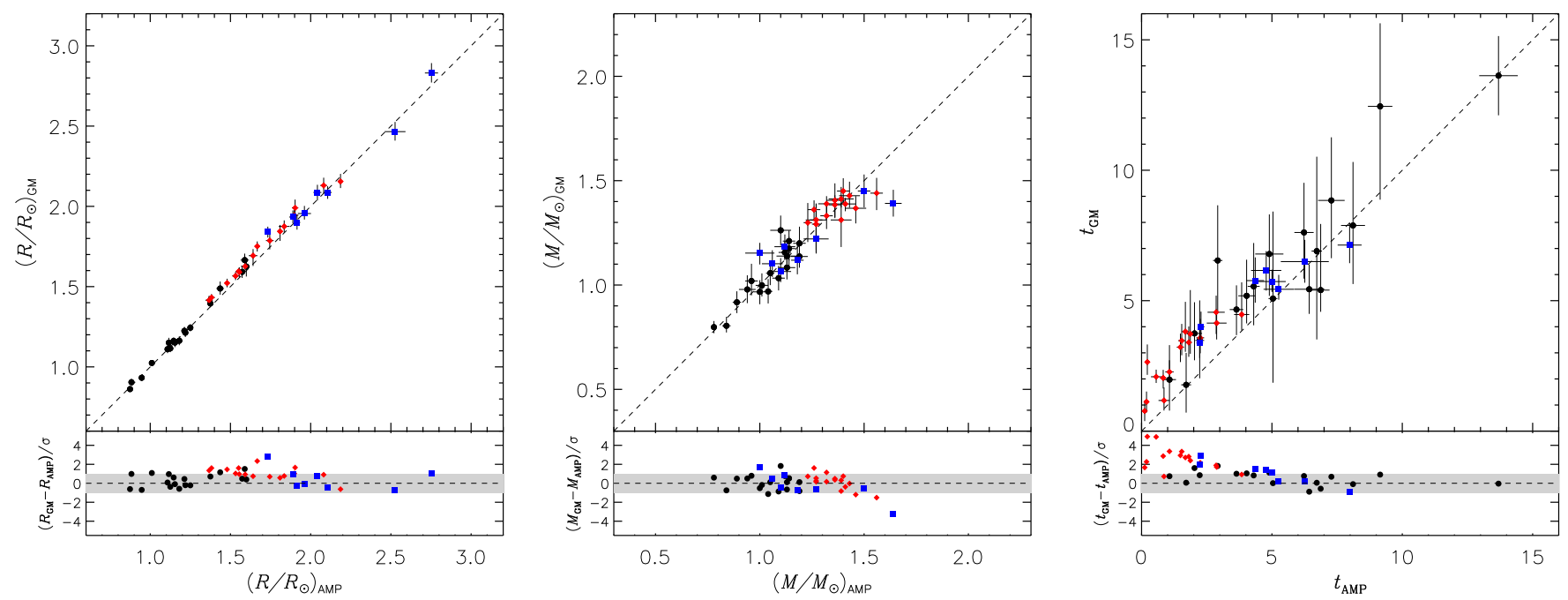

Figure 5. Comparison of the asteroseismic radii (left), masses (center), and ages (right) derived from grid-modeling (GM; Chaplin et al. 2014) with the AMP estimates from Table 1, including the simple (black circles), F-like (red diamonds), and mixed-mode stars (blue squares). The top panels compare the values and uncertainties, while the bottom panels show the differences between the estimates normalized by the uncertainty on the difference.

(A color version of this figure is available in the online journal.)

on the age - an improvement of a factor of two for the radius and $25 \%$ for the mass compared to the typical precision from scaling relations. The grid-modeling radii for F-like stars are systematically higher compared to AMP, but again the agreement is generally better than $2 \sigma$. For all three categories of stars, GARSTEC yields ages that are systematically older by $\sim 1$ Gyr for most targets with AMP ages below $\sim 3$ Gyr. Chaplin et al. (2014) noted this tendency of GARSTEC with respect to most of the other model grids they explored and attributed the offset to differences in the treatment of convective core overshoot, which is not included in the AMP models.

Modeling the individual frequencies with AMP led to a significant improvement in the internal precision of the derived stellar properties relative to estimates based on scaling relations or grid-modeling. The median uncertainties from AMP are 1.2\% on the radius, $2.8 \%$ on the mass, and $7.9 \%$ on the age-about a factor of three improvement over the radius and mass precision from scaling relations, and more precise than grid-modeling by about a factor of two in radius, mass, and age. It is more difficult to assess the absolute accuracy of the AMP results, but 12 stars in our sample (KIC 3632418, 3733735, 7747078, 8006161, 8228742, 9139151, 9139163, 9206432, 10162436, 10454113, 11253226, and 12258514) have a parallax from Hipparcos (van Leeuwen 2007), allowing us to compare the predicted luminosities with those of the AMP models that incorporate this constraint. Ten of the AMP luminosities are within $1 \sigma$ of the predictions, and only two stars show larger deviations ( $1.7 \sigma$ for 10162436, and $1.8 \sigma$ for 10454113) — fewer than expected 
for Gaussian distributed errors. One star (KIC 8006161) also has a radius from CHARA interferometry $\left(0.952 \pm 0.021 R_{\odot}\right.$; Huber et al. 2012), which is reproduced by the AMP model within $0.2 \sigma$ when the constraint was included and $2 \sigma$ when it was not. These subsamples suggest that, in addition to being more precise than other methods, the AMP results are also reasonably accurate.

\subsection{Applications of the Derived Stellar Properties}

The radii and masses in Table 1 provide a new opportunity to test the $v_{\max }$ scaling relation for main-sequence and subgiant stars (for previous discussion, see Stello et al. 2009; Bedding 2011; Belkacem et al. 2011, 2013; Huber et al. 2012). Such a test is meaningful because the AMP results were obtained without using the observed values of $v_{\max }$ as constraints. We consider the following form of the scaling relation (Equation (10) of Kjeldsen \& Bedding 1995):

$$
\frac{v_{\max }}{v_{\max , \odot}}=\frac{M / M_{\odot}}{\left(R / R_{\odot}\right)^{2} \sqrt{T_{\text {eff }} / T_{\text {eff }, \odot}}}
$$

with $v_{\max , \odot}=3090 \mu \mathrm{Hz}$ (Chaplin et al. 2014). Figure 6 compares the values of $v_{\max }$ calculated using Equation (6) with those measured for our sample by Chaplin et al. (2014). For about $70 \%$ of the stars the agreement is within $1 \sigma$, confirming the relation-although there is some evidence of systematic deviations that merits further investigation.

The improved estimates of stellar age in Table 1 allow us to refine the age-rotation-activity relations derived by Karoff et al. (2013). Among their sample of 20 stars that have been monitored for chromospheric activity since 2009 from the Nordic Optical Telescope, 17 appear in Table 1. The other three targets are KIC 4914923, which was not analyzed by Appourchaux et al. (2012); the spectroscopic binary KIC 8379927, which has contaminated atmospheric parameters; and the asteroseismic

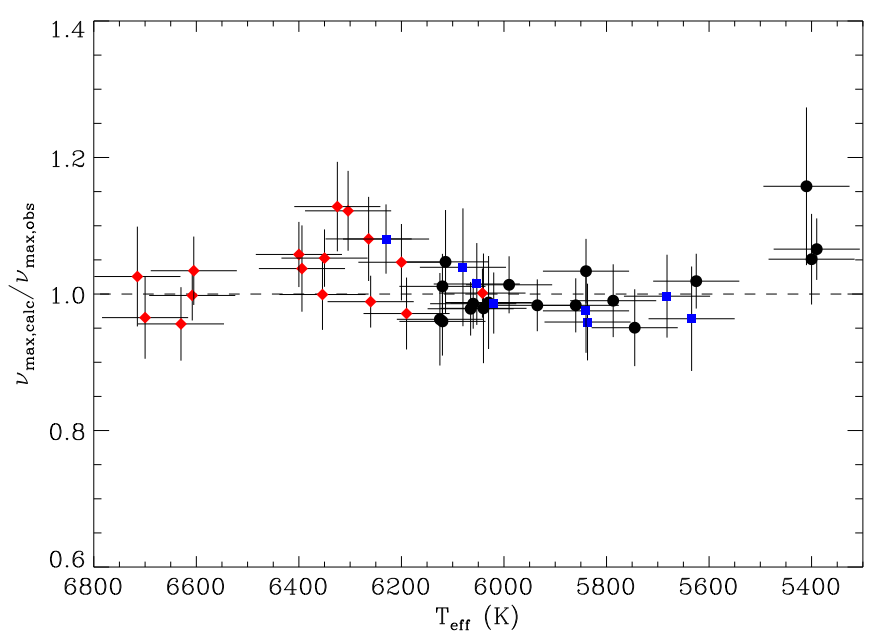

Figure 6. Ratio of $v_{\max }$ calculated from Equation (6) to that measured from the observed power spectrum (Chaplin et al. 2014) for simple (black circles), F-like (red diamonds), and mixed-mode stars (blue squares). Calculated values are derived from the radii and masses listed in Table 1 using the $T_{\text {eff }}$ values of Bruntt et al. (2012) with the errors from Chaplin et al. (2014).

(A color version of this figure is available in the online journal.)

binary KIC 10124866, which presents difficulties in extracting the overlapping oscillation spectra. Adopting the rotation periods $P_{\text {rot }}$ and net Ca II fluxes $\Delta \mathcal{F}_{\mathrm{Ca}}$ from Table 2 of Karoff et al. (2013), but replacing the grid-modeling ages from SEEK (Quirion et al. 2010) with the values from Table 1, we find

$$
\begin{gathered}
\log \Delta \mathcal{F}_{\mathrm{Ca}}=(-0.34 \pm 0.04) \log t+(5.94 \pm 0.02), \\
\log P_{\text {rot }}=(0.41 \pm 0.06) \log t+(0.75 \pm 0.03)
\end{gathered}
$$

The revised exponents on the age-activity and age-rotation relations (see Figure 7) differ significantly from the values

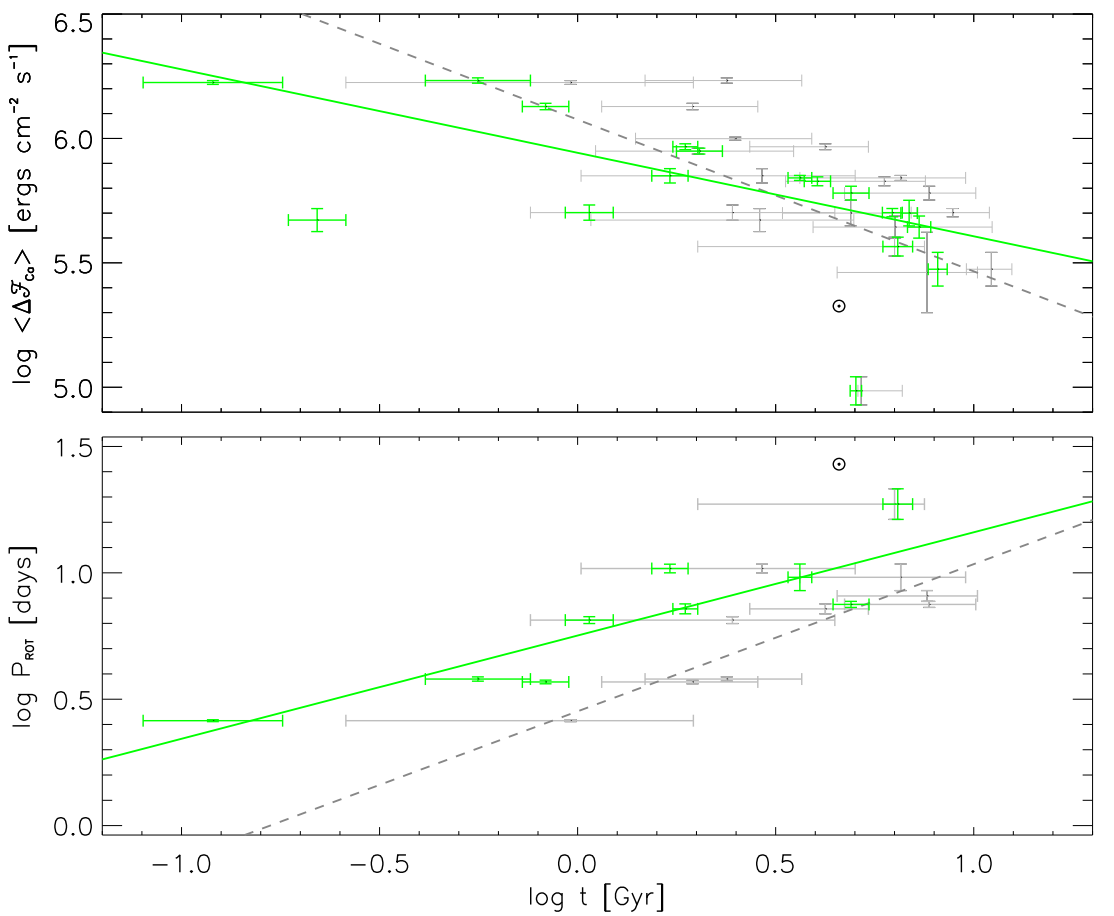

Figure 7. Age-activity (top; $\chi_{R}^{2}=247$ ) and age-rotation (bottom; $\chi_{R}^{2}=38$ ) relations from the Karoff et al. (2013) sample, using the original ages from SEEK (gray, dashed line) and the updated ages from AMP (green, solid line). Note that only SEEK results are shown for KIC 4914923, which is not included in our sample. The position of the Sun is indicated by the $\odot$ symbol.

(A color version of this figure is available in the online journal.) 


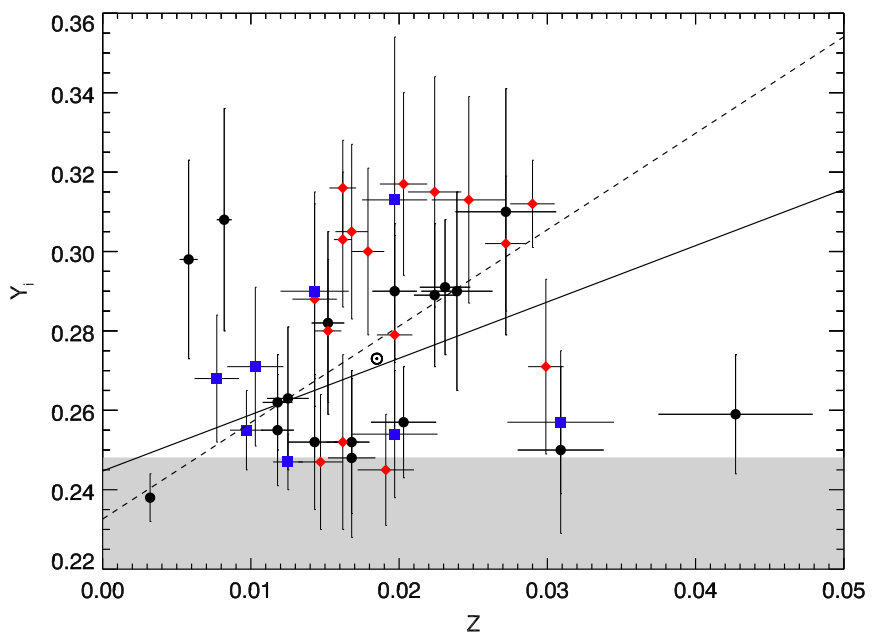

Figure 8. Linear least-squares fits for a relation between the metallicity $Z$ and the initial helium mass fraction $Y_{\mathrm{i}}$, considering errors only on the latter. Two linear fits are shown, one including all stars (solid line) and another excluding three outliers with $Z>0.03$ (dashed line). The shaded region denotes initial helium below the primordial value (Steigman 2010).

(A color version of this figure is available in the online journal.)

$(-0.61 \pm 0.17$ and $0.48 \pm 0.17)$ derived by Karoff et al. (2013), and from the original values of -0.54 and 0.51 found by Skumanich (1972). A more detailed analysis of this sample has recently been performed by Garcia et al. (2014).

The asteroseismic bulk composition can be used to test the predictions of Galactic chemical enrichment, from which we expect a linear relation between metallicity and initial helium with slope $\Delta Y / \Delta Z$. Observational and theoretical techniques have been used to determine this slope using $\mathrm{H}_{\text {II }}$ regions (Peimbert et al. 2007), eclipsing binaries (Ribas et al. 2000), and main-sequence broadening (Casagrande et al. 2007), with results ranging from 1.0 to 2.5 . The determination of stellar helium abundance is an inherently difficult problem, so it is important to consider the results from a variety of methods, including asteroseismic analysis. The least-squares linear fit to all stars is $Y_{\mathrm{i}}=(1.42 \pm 0.27) Z+(0.245 \pm 0.005)$, with $\chi_{R}^{2}=$ 2.2. Excluding a few high-metallicity outliers (KIC 3424541 , 6603624 and 8006161), the relation becomes $Y_{\mathrm{i}}=(2.43 \pm$ $0.34) Z+(0.233 \pm 0.005)$ with $\chi_{R}^{2}=1.7$. The slopes of these two fits span nearly the full range of other determinations of $\Delta Y / \Delta Z$, and the scatter around either relation is rather large-for a given $Z$, our values of $Y_{\mathrm{i}}$ show deviations of more than \pm 0.03 (see Figure 8). ${ }^{39}$ A more direct determination of helium abundances in stellar envelopes, e.g., using acoustic glitches (Mazumdar et al. 2014; Verma et al. 2014), would be very helpful as an independent constraint for global model-fitting.

We can compare our estimates of the mixing-length $\alpha$ and the amplitude of the surface correction $a_{0}$ to recent 3D simulations of convective stellar atmospheres at solar metallicity (Trampedach et al. 2013, 2014). The values of $\alpha$ in our sample decrease slightly with $T_{\text {eff }}$, and a tri-linear regression also reveals small increases with both gravity and metallicity. Although the AMP values exhibit a larger range, the trends with $T_{\text {eff }}$ and gravity qualitatively agree with the latest calibration of $\alpha$ (Trampedach et al. 2014), while the trend with metallicity has not yet been sampled by those simulations. The 3D simulations

\footnotetext{
39 Note that one value of $Y_{\mathrm{i}}$ is significantly below the primordial helium abundance, while three others are marginally below $Y_{\mathrm{P}}$. Recall that heavy element diffusion and settling was not included in our stellar evolution models.
}

also predict a convective expansion of the atmosphere relative to one-dimensional (1D) models-an important contribution to the surface term $a_{0}$. The expansion increases monotonically with higher $T_{\text {eff }}$ and lower $\log g$ (Trampedach et al. 2013), but in 1D models it is strongly coupled to the value of $\alpha$, which controls the slope of the temperature gradient near the top of the convection zone. Physical constraints on $\alpha$ and physical modeling of the surface effect from future 3D simulations promise to improve the reliability and robustness of asteroseismic fitting.

\section{DISCUSSION}

Our uniform asteroseismic analysis for a large sample of Kepler main-sequence and subgiant stars yielded precise determinations of many stellar properties (see Table 1), and some important lessons for future work. Most of the simple stars have values of $f \leqslant 10$, indicating a reasonable match between the models from AMP and the observational constraints. The exceptions (KIC 6603624, 8694723, 8760414, and 11244118) are stars with the most extreme metallicities in our sample, which may require modifications to the solar-composition mixture in the opacity tables employed by AMP for more accurate modeling. More of the F-like stars have $f>10$, but these are generally targets where the $\ell=0$ and $\ell=2$ modes are most difficult to separate. The échelle diagrams in Figure 3 show that the extracted frequencies for F-like stars from Appourchaux et al. (2012) include variations in the small separations $d_{0,2}(n)$ that cannot be reproduced by models-in which the $\ell=0,2$ ridges always curve together with only monotonic variations in $d_{0,2}(n)$. This suggests that the smoothness criteria in peakbagging pipelines may need to be modified for F-like stars. Automated fitting of the mixed-mode stars is limited to those with fewer than $\sim 3$ avoided crossings and the match to the observed frequencies is generally less precise, with most stars showing $f>10$. The greatest difficulties are again encountered for extreme metallicity targets (KIC 7976303 and 10018963), but the qualitative agreement with the locations of avoided crossings for most of the mixed-mode stars in Figure 3 is remarkable. Ultraprecise constraints on the properties of these stars must rely on future dense grid-modeling to refine the estimates from Table 1.

Our modifications to the fitting procedures to try and avoid a bias toward low-helium solutions have helped, but the problem was not eliminated entirely. As noted in Section 3.1, of the 22 stars considered by Mathur et al. (2012) 6 of them (27\%) yielded an initial helium mass fraction significantly below the primordial value, while four additional targets (18\%) were marginally below $Y_{\mathrm{P}}$. We attempted to address this issue by including the frequency ratios as additional constraints, and by adopting larger uncertainties at higher frequencies where the surface correction is larger. A smaller fraction of our sample of 42 stars appears to be affected by the low-helium bias, but Table 1 still includes one star $(2.4 \%)$ with $Y_{\mathrm{i}}$ significantly below $Y_{\mathrm{P}}$ and three additional targets $(7.1 \%)$ that fall marginally below $Y_{\mathrm{P}}$. Further improvement may require an alternative surface correction (e.g., Christensen-Dalsgaard 2012).

The precision of asteroseismic data sets from extended observations with Kepler now demands that we address the dominant sources of systematic error in the stellar models. The largest source arises from incomplete modeling of the nearsurface layers. Although something like the empirical correction of Kjeldsen et al. (2008) will continue to be useful, we are now in a position to capture more of the relevant physics. For example, Gruberbauer et al. (2013) performed a Bayesian analysis of the 22 Kepler targets from Mathur et al. (2012), including a 
simplified non-adiabatic treatment of the pulsations. They found that the Bayesian probabilities were higher when non-adiabatic rather than adiabatic frequencies were fit to the observations, and that for most stars the surface effect was minimized and in some cases even eliminated. Their non-adiabatic model accounted for radiative losses and gains but neglected perturbations to the convective flux and turbulent pressure (Guenther 1994). In the case of the Sun, the stability and frequency of the oscillation modes depends substantially on turbulent pressure and the inclusion of non-local effects in the treatment of convection (Balmforth 1992a, 1992b; Houdek 2010), and the resulting frequency shift is uncertain. The sensitivity of the results to the model of convection and the temperature profile in the super-adiabatic layer was also emphasized by Rosenthal et al. (1999) and Li et al. (2002). Regardless, the Bayesian approach has the advantage of incorporating the unknown sources of systematic error directly into the uncertainties on the derived stellar properties, and can reveal which approach to the pulsation calculations generally improves the model fits.

For future analyses, we intend to augment ADIPLS with the non-adiabatic stellar oscillation code GYRE (Townsend \& Teitler 2013), which currently includes a limited treatment of non-adiabatic effects but is flexible enough to incorporate additional contributions. We would also like to take advantage of the numerical stability and modular architecture of the opensource MESA code (Paxton et al. 2013) to explore different chemical mixtures and to include heavy element diffusion and settling in the evolutionary models, which is not currently stable for all types of stars with ASTEC. With these new modules for stellar evolution and pulsation calculations, we can embed a Bayesian formalism into the parallel genetic algorithm to complement the simple $\chi^{2}$ approach. The complete sample of asteroseismic targets from extended Kepler observations spanning up to four years will provide a rich data set to validate these new ingredients for the next generation of AMP.

The golden age of asteroseismology for main-sequence and subgiant stars owes a great debt to the Kepler mission, but it promises to continue with the anticipated launch of NASA's Transiting Exoplanet Survey Satellite (TESS; Ricker et al. 2014) in 2017. While Kepler was able to provide asteroseismic data for hundreds of targets and could simultaneously monitor 512 stars with 1-minute sampling, TESS plans to observe $\sim 500,000$ of the brightest G- and K-type stars in the sky at a cadence sufficient to detect solar-like oscillations. The data sets will be nearly continuous for at least 27 days, but in two regions near the ecliptic poles the fields will overlap for durations up to a full year. These brighter stars will generally be much better characterized than the Kepler targets-with parallaxes from Hipparcos and ultimately Gaia (Perryman et al. 2001), and reliable atmospheric constraints from ground-based spectroscopy-making asteroseismic characterization more precise and accurate. With several years of development time available, AMP promises to be ready to convert this avalanche of data into reliable inferences on the properties of our solar system's nearest neighbors.

We would like to thank Victor Silva Aguirre for helpful discussions. This work was supported in part by NASA grants NNX13AC44G and NNX13AE91G, and by White Dwarf Research Corporation through the Pale Blue Dot project (http://whitedwarf.org/palebluedot/). Computational time on Kraken at the National Institute of Computational Sciences was provided through XSEDE allocation TG-AST090107. Funding for the Stellar Astrophysics Centre is provided by The
Danish National Research Foundation (grant DNRF106). We acknowledge the ASTERISK project (ASTERoseismic Investigations with SONG and Kepler) funded by the European Research Council (grant agreement No.: 267864), the Scientific and Technological Research Council of Turkey (TÜBITAK:112T989), and a European Commission grant for the SPACEINN project (FP7-SPACE-2012-312844). B.P.B. was supported in part by NSF Astronomy and Astrophysics postdoctoral fellowship AST 09-02004. C.M.S.O. is supported by NSF grant PHY 08-21899 and K.I.T.P. is supported by NSF grant PHY 11-25915. M.S.C. is supported by an Investigador FCT contract funded by FCT/MCTES (Portugal) and POPH/FSE (EC). A.D. has been supported by the Hungarian OTKA grants K83790, KTIA URKUT_10-1-2011-0019 grant, the Lendület-2009 Young Researchers Programme of the Hungarian Academy of Sciences, the János Bolyai Research Scholarship of the Hungarian Academy of Sciences and the City of Szombathely under agreement No. S-11-1027. A.D. and R.A.G. acknowledge the support of the European Community Seventh Framework Programme (FP7/2007-2013) under grant agreement No. 269194 (IRSES/ASK). R.A.G. and D. Salabert acknowledge the support of the CNES grant at CEA-Saclay. A.M. acknowledges support from the NIUS programme of HBCSE (TIFR). A.S. is supported by the MICINN grant AYA201124704 and by the ESF EUROCORES Program EuroGENESIS (MICINN grant EUI2009-04170). D. Stello is supported by the Australian Research Council.

\section{APPENDIX}

\section{UNCERTAINTY ESTIMATION PROCEDURE}

Our previous approach to uncertainty estimation using SVD is no longer appropriate for several reasons. First, SVD assumes that the observables are independent. This was reasonable when we were only fitting the individual frequencies and spectroscopic constraints. Now that we also fit the frequency ratios $r_{02}$ and $r_{010}$ (see Section 3.2), some of the observables are no longer independent and SVD cannot be used. Second, even if we do not include the frequency ratios as constraints, the SVD method determines the uncertainties from the local shape of the $\chi^{2}$ surface. Such an analysis fails to capture the uncertainties due to non-uniqueness of the optimal solution, yielding errors that are too optimistic for practical purposes. Finally, the metric used by the GA for optimization, which defines the local shape of the $\chi^{2}$ surface, is a composite of normalized $\chi^{2}$ values from several distinct sets of observables, making it difficult to interpret in a statistically robust manner.

We can use the ensemble of models sampled by the GA to provide a more conservative and global estimate of the uncertainties. During an AMP run, the parameter values and average $\chi^{2}$ metric are recorded for each trial model that is compared to the observations. The nature of the fitting process ensures that each trial model is a much better match to the observations than a random model in the search space. For each stellar evolution track generated by AMP, the age is optimized internally using a binary decision tree to match the observed large separation. In addition, the final age is interpolated between time steps on the track to match the lowest observed radial mode, and the empirical surface correction of Kjeldsen et al. (2008) is applied to improve the match to higher frequency modes. In effect, the GA is producing the best possible match to the observations given the four fixed parameters $\left(M, Z, Y_{\mathrm{i}}, \alpha\right)$ for each trial model. As a consequence, we need to use a subset 

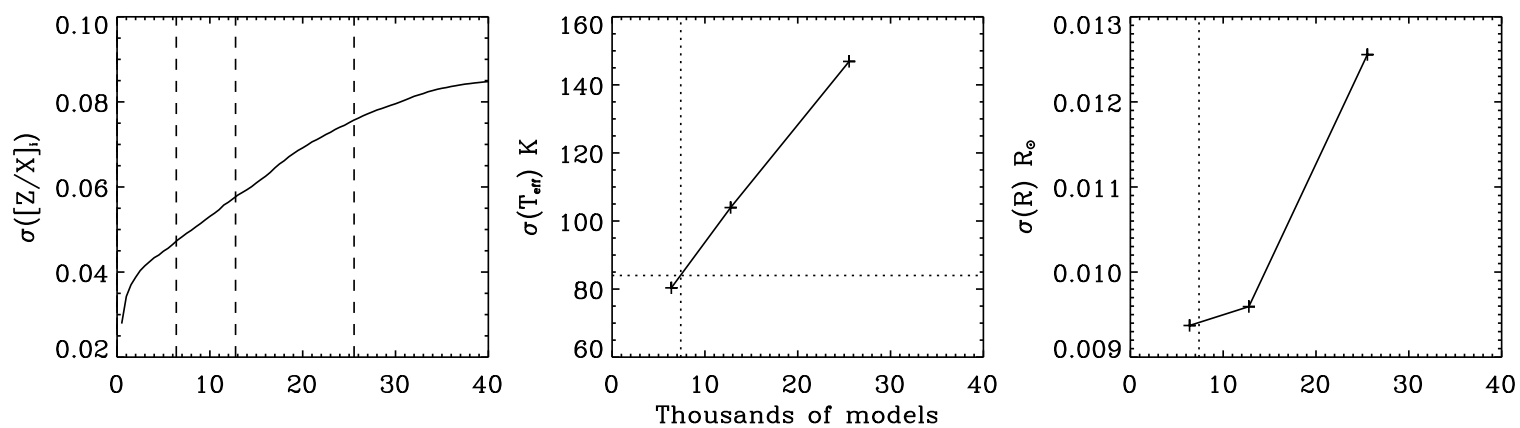

Figure 9. Uncertainties in $[Z / X]_{\mathrm{i}}$ (left), $T_{\mathrm{eff}}$ (center), and $R$ (right) for KIC 6116048 as the number of models included in the likelihood-weighted mean is increased. Several cuts are defined from the uncertainty in $[Z / X]_{\mathrm{i}}$ (left panel, vertical dashed lines), which yield corresponding uncertainties in $T_{\text {eff }}$ and $R$ from the $1 \sigma$ models (center and right panels, + points). Interpolating to yield an uncertainty of $84 \mathrm{~K}$ in $T_{\text {eff }}$ (center panel, horizontal dotted line) provides the final cut (center and right panels, vertical dotted line) that is used to define the uncertainties on all model properties.

of the full ensemble of GA models if we want a reasonable estimate of the uncertainties from the limited information that is available.

To determine the number of GA trial models that we should include in our uncertainty estimation procedure, we initially attempted to match the observational error on $[\mathrm{M} / \mathrm{H}](0.09$ dex; Chaplin et al. 2014; Bruntt et al. 2012). As outlined briefly in Section 4.1, we ranked the unique trial models by their average $\chi^{2}$ value and assigned each one a relative likelihood using Equation (5). This allowed us to calculate a likelihoodweighted mean and standard deviation for each adjustable model parameter, including $Z$ and $Y_{\mathrm{i}}$ to generate an uncertainty on the initial composition $[Z / X]_{i}$ as we gradually included more models in the mean. We only had access to the initial value of $X$, which increases at the surface over time as helium diffusion and settling operates in the models, so this only provided a first estimate of the appropriate number of models to include. The resulting uncertainty on $[Z / X]_{\mathrm{i}}$ for our example star KIC 6116048 is shown in the left panel of Figure 9 as a function of the number of models included in the likelihood-weighted mean.

Uncertainties on the other properties of the optimal models can only be determined after a cut on the number of models has been adopted. The cut establishes the uncertainties on the adjustable parameters $\left(M, t, Z, Y_{\mathrm{i}}, \alpha\right)$ as well as the covariance matrix around the optimal solution. This allows us to calculate a set of models that define the $1 \sigma$ error ellipse, and then use half the range of values for any other property (such as $[Z / X]_{\mathrm{s}}$, $T_{\text {eff }}$, or $R$ ) within the $1 \sigma$ models to define an uncertainty for these non-adjustable parameters. For KIC 6116048, we determined that by including the best $\sim 25,000$ models in the likelihood-weighted mean, the resulting uncertainty on the surface composition $[Z / X]_{\mathrm{s}}$ (not shown in Figure 9) was comparable to the observational error on $[\mathrm{M} / \mathrm{H}]$. Using this same cut, the full range of model values for $T_{\text {eff }}$ within the $1 \sigma$ models spanned $290 \mathrm{~K}$, yielding an error estimate of $\pm 145 \mathrm{~K}$ (see center panel of Figure 9). We repeated the analysis procedure with a cut at one-half and one-quarter of this number of models (vertical dashed lines in the left panel of Figure 9) until the resulting uncertainty on the model $T_{\text {eff }}$ was below the error on the observed $T_{\text {eff }}$ (84 K; Chaplin et al. 2014; Bruntt et al. 2012).

By interpolating the number of models required to reproduce the observed $T_{\text {eff }}$ error, we defined the appropriate cut that was then used to estimate the final uncertainties on all model properties. The center panel of Figure 9 shows the uncertainty on the model $T_{\text {eff }}$ for the three cuts indicated in the left panel.
When the 7370 best models sampled by the GA (vertical dotted line) were used to calculate the likelihood-weighted mean and standard deviation for each of the adjustable model parameters, the uncertainty on the model $T_{\text {eff }}$ from the resulting $1 \sigma$ models was equal to the observational error of $84 \mathrm{~K}$ (horizontal dotted line). The range of radii within this same set of $1 \sigma$ models define the radius uncertainty of $0.0094 R_{\odot}$ (right panel of Figure 9). As noted in Section 4.1, the above procedure yields inherently conservative uncertainty estimates because it assumes that the asteroseismic data do not contribute to the determination of $T_{\text {eff }}$ in the final solution. This assumption is certainly not valid for the surface composition, even ignoring the complications from diffusion, which explains why the final uncertainty on $[Z / X]_{\mathrm{i}}$ is well below the observational error on $[\mathrm{M} / \mathrm{H}]$. We repeated the above procedure for each star in our sample to yield the final set of uncertainties, which appear in Table 1.

\section{REFERENCES}

Aizenman, M., Smeyers, P., \& Weigert, A. 1977, A\&A, 58, 41

Alexander, D. R., \& Ferguson, J. W. 1994, ApJ, 437, 879

Ammons, S. M., Robinson, S. E., Strader, J., et al. 2006, ApJ, 638, 1004

Angulo, C., Arnould, M., Rayet, M., et al. 1999, NuPhA, 656, 3

Appourchaux, T., Chaplin, W. J., García, R. A., et al. 2012, A\&A, 543, A54

Appourchaux, T., Michel, E., Auvergne, M., et al. 2008, A\&A, 488, 705

Bahcall, J. N., Pinsonneault, M. H., \& Wasserburg, G. J. 1995, RvMP, 67, 781

Balmforth, N. J. 1992a, MNRAS, 255, 632

Balmforth, N. J. 1992b, MNRAS, 255, 639

Bedding, T. R. 2011, Asteroseismology: XXII Canary Islands Winter School of Astrophysics, ed. P. L. Pallé (Cambridge: Cambridge Univ. Press), 60

Bedding, T. R., \& Kjeldsen, H. 2003, PASA, 20, 203

Belkacem, K., Goupil, M. J., Dupret, M. A., et al. 2011, A\&A, 530, A142

Belkacem, K., Samadi, R., Mosser, B., Goupil, M.-J., \& Ludwig, H.-G. 2013, in ASP Conf. Ser. 479, Progress in Physics of the Sun and Stars: A New Era in Helio- and Asteroseismology, ed. H. Shibahashi \& A. E. Lynas-Gray (San Francisco, CA: ASP), 61

Benomar, O., Bedding, T. R., Mosser, B., et al. 2013, ApJ, 767, 158

Bevington, P. R., \& Robinson, D. K. 1992, Data Reduction and Error Analysis for the Physical Sciences (New York: McGraw-Hill)

Böhm-Vitense, E. 1958, ZAp, 46, 108

Borucki, W. J., Koch, D., Basri, G., et al. 2010, Sci, 327, 977

Brandão, I. M., Doğan, G., Christensen-Dalsgaard, J., et al. 2011, A\&A, 527, A37

Brown, T. M., Gilliland, R. L., Noyes, R. W., \& Ramsey, L. W. 1991, ApJ, 368,599

Brown, T. M., Latham, D. W., Everett, M. E., \& Esquerdo, G. A. 2011, AJ, 142,112

Bruntt, H., Basu, S., Smalley, B., et al. 2012, MNRAS, 423, 122

Casagrande, L., Flynn, C., Portinari, L., Girardi, L., \& Jimenez, R. 2007, MNRAS, 382, 1516

Chaplin, W. J., Basu, S., Huber, D., et al. 2014, ApJS, 210, 1

Chaplin, W. J., Kjeldsen, H., Christensen-Dalsgaard, J., et al. 2011, Sci, 332,213 
Chaplin, W. J., \& Miglio, A. 2013, ARA\&A, 51, 353

Christensen-Dalsgaard, J. 2008a, Ap\&SS, 316, 13

Christensen-Dalsgaard, J. 2008b, Ap\&SS, 316, 113

Christensen-Dalsgaard, J. 2012, AN, 333, 914

Christensen-Dalsgaard, J., Dappen, W., Ajukov, S. V., et al. 1996, Sci, 272, 1286

Creevey, O. L. 2009, in ASP Conf. Ser. 416, Solar-Stellar Dynamos as Revealed by Helio- and Asteroseismology: GONG 2008/SOHO 21, ed. M. Dikpati, T. Arentoft, I. G. Hernández, C. Lindsey, \& F. Hill (San Francisco, CA: ASP), 363

Creevey, O. L., Doǧan, G., Frasca, A., et al. 2012, A\&A, 537, A111

Deheuvels, S., \& Michel, E. 2011, A\&A, 535, A91

Doǧan, G., Metcalfe, T. S., Deheuvels, S., et al. 2013, ApJ, 763, 49

Ferguson, J. W., Alexander, D. R., Allard, F., et al. 2005, ApJ, 623, 585

Flower, P. J. 1996, ApJ, 469, 355

Frasca, A., Alcalá, J. M., Covino, E., et al. 2003, A\&A, 405, 149

Frasca, A., Guillout, P., Marilli, E., et al. 2006, A\&A, 454, 301

Garcia, R. A., Ceillier, T., Salabert, D., et al. 2014, A\&A, submitted (arXiv:1403.7155)

García, R. A., Hekker, S., Stello, D., et al. 2011, MNRAS, 414, L6

Gilliland, R. L., Jenkins, J. M., Borucki, W. J., et al. 2010, ApJL, 713, L160

Goldreich, P., \& Keeley, D. A. 1977, ApJ, 212, 243

Goldreich, P., \& Kumar, P. 1988, ApJ, 326, 462

Grec, G., Fossat, E., \& Pomerantz, M. 1980, Natur, 288, 541

Grevesse, N., \& Noels, A. 1993, Perfectionnement de l'Association Vaudoise des Chercheurs en Physique, 205

Gruberbauer, M., Guenther, D. B., MacLeod, K., \& Kallinger, T. 2013, MNRAS, 435,242

Gruberbauer, M., Kallinger, T., Weiss, W. W., \& Guenther, D. B. 2009, A\&A, 506, 1043

Guenther, D. B. 1994, ApJ, 422, 400

Guenther, D. B., \& Brown, K. I. T. 2004, ApJ, 600, 419

Houdek, G. 2010, AN, 331, 998

Houdek, G., Balmforth, N. J., Christensen-Dalsgaard, J., \& Gough, D. O. 1999, A\&A, 351, 582

Huber, D., Ireland, M. J., Bedding, T. R., et al. 2012, ApJ, 760, 32

Iglesias, C. A., \& Rogers, F. J. 1996, ApJ, 464, 943

Karoff, C., Metcalfe, T. S., Chaplin, W. J., et al. 2013, MNRAS, 433, 3227

Kjeldsen, H., \& Bedding, T. R. 1995, A\&A, 293, 87

Kjeldsen, H., Bedding, T. R., \& Christensen-Dalsgaard, J. 2008, ApJL, 683, L175

Kjeldsen, H., Christensen-Dalsgaard, J., Handberg, R., et al. 2010, AN, 331,966

Lebreton, Y., \& Goupil, M.-J. 2014, A\&A, 569, 21

Li, L. H., Robinson, F. J., Demarque, P., Sofia, S., \& Guenther, D. B. 2002, ApJ, 567,1192

Mathur, S., Hekker, S., Trampedach, R., et al. 2011, ApJ, 741, 119

Mathur, S., Metcalfe, T. S., Woitaszek, M., et al. 2012, ApJ, 749, 152
Mazumdar, A., Monteiro, M. J. P. F. G., Ballot, J., et al. 2014, ApJ, 782, 18 Metcalfe, T. S., Chaplin, W. J., Appourchaux, T., et al. 2012, ApJL, 748, L10 Metcalfe, T. S., \& Charbonneau, P. 2003, JCoPh, 185, 176

Metcalfe, T. S., Creevey, O. L., \& Christensen-Dalsgaard, J. 2009, ApJ, 699,373

Metcalfe, T. S., Monteiro, M. J. P. F. G., Thompson, M. J., et al. 2010, ApJ, 723,1583

Michaud, G., \& Proffitt, C. R. 1993, in Proc. IAU Colloq. 137, Inside the Stars, ed. A. Baglin \& W. W. Weiss (ASP Conf. Ser. 40; San Francisco, CA: ASP), 246

Molenda-Żakowicz, J., Sousa, S. G., Frasca, A., et al. 2013, MNRAS, 434, 1422

Osaki, J. 1975, PASJ, 27, 237

Paxton, B., Cantiello, M., Arras, P., et al. 2013, ApJS, 208, 4

Peimbert, M., Luridiana, V., \& Peimbert, A. 2007, ApJ, 666, 636

Perryman, M. A. C., de Boer, K. S., Gilmore, G., et al. 2001, A\&A, 369, 339

Pinsonneault, M. H., An, D., Molenda-Żakowicz, J., et al. 2012, ApJS, 199, 30

Quirion, P.-O., Christensen-Dalsgaard, J., \& Arentoft, T. 2010, ApJ, 725, 2176

Ribas, I., Jordi, C., Torra, J., \& Giménez, Á. 2000, MNRAS, 313, 99

Ricker, G. R., et al. 2014, SPIE Proceedings, submitted (arXiv:1406.0151)

Rogers, F. J., \& Nayfonov, A. 2002, ApJ, 576, 1064

Rosenthal, C. S., Christensen-Dalsgaard, J., Nordlund, Å., Stein, R. F., \& Trampedach, R. 1999, A\&A, 351, 689

Roxburgh, I. W., \& Vorontsov, S. V. 2003, A\&A, 411, 215

Samadi, R., \& Goupil, M.-J. 2001, A\&A, 370, 136

Silva Aguirre, V., Basu, S., Brandão, I. M., et al. 2013, ApJ, 769, 141

Skumanich, A. 1972, ApJ, 171, 565

Sneden, C. A. 1973, PhD thesis, Univ. of Texas

Sousa, S. G., Santos, N. C., Israelian, G., Mayor, M., \& Monteiro, M. J. P. F. G. 2007, A\&A, 469, 783

Steigman, G. 2010, JCAP, 4, 29

Stello, D., Chaplin, W. J., Basu, S., Elsworth, Y., \& Bedding, T. R. 2009, MNRAS, 400, L80

Tassoul, M. 1980, ApJS, 43, 469

Torres, G. 2010, AJ, 140, 1158

Torres, G., Fischer, D. A., Sozzetti, A., et al. 2012, ApJ, 757, 161

Townsend, R. H. D., \& Teitler, S. A. 2013, MNRAS, 435, 3406

Trampedach, R., Asplund, M., Collet, R., Nordlund, Å., \& Stein, R. F. 2013, ApJ 769,18

Trampedach, R., Christensen-Dalsgaard, J., Nordlund, Å., Asplund, M., \& Stein, R. F. 2014, MNRAS, 442, 805

van Leeuwen, F. 2007, A\&A, 474, 653

Verma, K., Faria, J. P., Antia, H. M., et al. 2014, ApJ, 790, 138

Verner, G. A., Elsworth, Y., Chaplin, W. J., et al. 2011, MNRAS, 415, 3539

Weiss, A., \& Schlattl, H. 2008, Ap\&SS, 316, 99

White, T. R., Bedding, T. R., Gruberbauer, M., et al. 2012, ApJL, 751, L36

Woitaszek, M., Metcalfe, T., \& Shorrock, I. 2009, Proc. of the 5th Grid Computing Environments Workshop (New York: ACM Press), 1 